ARTICLE

https://doi.org/10.1038/s41467-019-08317-8

\title{
Mechanism of IRSp53 inhibition by 14-3-3
}

\author{
David J. Kast (i) ${ }^{1,2} \&$ Roberto Dominguez (i) ${ }^{1}$
}

Filopodia are precursors of dendritic spines and polarized cell migration. The I-BAR-domain protein IRSp53 is a key regulator of filopodia dynamics that couples Rho-GTPase signaling to cytoskeleton and membrane remodeling, playing essential roles in neuronal development and cell motility. Here, we describe the structural-functional basis for 14-3-3-dependent inhibition of IRSp53. Phosphoproteomics, quantitative binding and crystallographic studies demonstrate that 14-3-3 binds to two pairs of phosphorylation sites in IRSp53. Using bicistronic expression, we obtain an IRSp53 heterodimer in which only one subunit is phosphorylated, and show that each subunit of IRSp53 independently binds one 14-3-3 dimer. A FRET-sensor assay using natively phosphorylated IRSp53 reveals opposite conformational changes upon binding of activatory (Cdc42, Eps8) or inhibitory (14-3-3) inputs. Finally, we show that 14-3-3 inhibits IRSp53 binding to membranes. Collectively, our findings support a mechanism whereby phosphorylation-dependent inhibition of IRSp53 by 14-3-3 counters membrane binding and interactions with $\mathrm{Cdc} 42$ and downstream cytoskeletal effectors.

\footnotetext{
${ }^{1}$ Department of Physiology, Perelman School of Medicine, University of Pennsylvania, Philadelphia, PA 19104, USA. ${ }^{2}$ Present address: Department of Cell Biology and Physiology, Washington University, St. Louis, MO 63110, USA. Correspondence and requests for materials should be addressed to R.D. (email: droberto@pennmedicine.upenn.edu)
} 
tight correlation between plasma membrane and actin cytoskeleton dynamics is a common feature of many cellular functions, including cell migration, organelle trafficking and endo/exocytosis. Bin/Amphiphysin/Rvs (BAR) domain proteins are essential spatio-temporal coordinators of signaling events to actin cytoskeleton and membrane dynamics. The superfamily of BAR domain proteins comprises a diverse group of multi-functional effectors, containing $\mathrm{N}$ - and/or Cterminal to the membrane-binding BAR domain additional signaling and protein-protein or protein-membrane interaction modules, including $\mathrm{SH} 3, \mathrm{PX}, \mathrm{PH}, \mathrm{RhoGEF}$, and RhoGAP domains ${ }^{1,2}$. Depending on the shape of the BAR domain, three subfamilies are distinguished: the canonical crescent-shaped BAR, the more extended and less curved F-BAR, and the inverse curvature I-BAR subfamilies. The most prominent member of the IBAR subfamily is IRSp53 (also known as BAIAP2), a protein implicated in the formation of plasma membrane protrusions such as filopodia ${ }^{3-10}$ and dendritic spines ${ }^{11-16}$. IRSp53 functions under the control of $\mathrm{Cdc} 42^{4,6,9,10,17,18}$, and recruits to the plasma membrane a long list of cytoskeletal effectors and synaptic scaffolds, including Eps8 $8^{6,19}$, Ena/VASP ${ }^{4,9,20}$, N-WASP ${ }^{18}$, WAVE $^{3,21,22}, \mathrm{mDia}^{8,23}, \mathrm{PSD}-95^{11,12,24,25}$, and Shank3 ${ }^{11,17}$. As a consequence, IRSp53 is both a key player in normal developmental processes, such as dendritic spine development ${ }^{16}$, myoblast fusion ${ }^{26}$, and eye development ${ }^{27}$, as well as a frequent factor in diseases, including tumorigenesis ${ }^{19,28}$ and neurological disorders ranging from autism spectrum disorder ${ }^{29}$ and schizophrenia $^{30}$ to attention deficit hyperactivity disorder ${ }^{31}$.

IRSp53 features an N-terminal I-BAR domain (residues 1-231) implicated in membrane binding ${ }^{32-34}$. The I-BAR domain is immediately followed by a partial CRIB motif, which is interrupted by a proline-rich sequence and is thus referred to as the CRIB-PR domain (residues 260-291) ${ }^{10}$. C-terminal to the CRIBPR domain, and connected by an 85 -amino acid linker rich in serine, threonine, and proline residues, IRSp53 presents an SH3 domain (residues 375-437). The SH3 domain mediates most of the interactions of IRSp53 with downstream cytoskeleton effectors. In the inactive state, the $\mathrm{SH} 3$ domain binds intramolecularly to the CRIB-PR domain, resulting in a compact, closed conformation ${ }^{10}$. A transition toward an active, open conformation can be triggered by the binding of either Cdc42 to the CRIB-PR domain or cytoskeletal effectors to the $\mathrm{SH} 3$ domain $^{10}$. Cdc42dependent activation of IRSp53 in cells results in increased formation of membrane ruffles and filopodia-like structures $^{4,6,9,10,17,18}$. The region between the CRIB-PR and SH3 domains hosts multiple phosphorylation sites, and some of these sites have been implicated in 14-3-3 binding ${ }^{35,36}$, which can lead to loss of cell polarity ${ }^{35}$.

Here, we show that phosphorylation of two pairs of sites within this region triggers the binding of 14-3-3 to IRSp53, which inhibits membrane binding and the interactions of $\mathrm{Cdc} 42$ with the CRIB-PR domain and cytoskeletal effectors with the $\mathrm{SH} 3$ domain.

\section{Results}

Purification of 14-3-3-binding-competent IRSp53. Mammals express seven 14-3-3 isoforms, denoted $\beta, \gamma, \varepsilon, \zeta, \eta, \sigma$, and $\theta$ (also called $\tau$ ), and most isoforms are enriched in brain tissue ${ }^{37}$, where IRSp53 is also abundant ${ }^{7,16}$. IRSp53 has been shown to interact in vitro and in cells with several 14-3-3 isoforms, including $\zeta, \gamma, \theta$, and $\sigma^{35,36,38,39}$. Here, we established conditions that enhance the amount of IRSp53 that binds 14-3-3 in mammalian cells, and developed a protocol for the purification of this protein in large quantities for biochemical and phosphoproteomics studies (Fig. 1). A previous report linked IRSp53 phosphorylation at T340 and T360 to the binding of $14-3-3^{36}$. These authors additionally found that IRSp53 coimunoprecipitates less abundantly with 14-3-3 when cells are treated with $\mathrm{LiCl}$, leading them to suggest that a kinase downstream of GSK3 $\beta$ phosphorylates IRSp53 at 14-3-3-binding sites. However, another study found that a Par1b/MARK2 mutant that accepts $N^{6}$-benzyl-ATP $\left[{ }^{35} \gamma \mathrm{S}\right]$ a

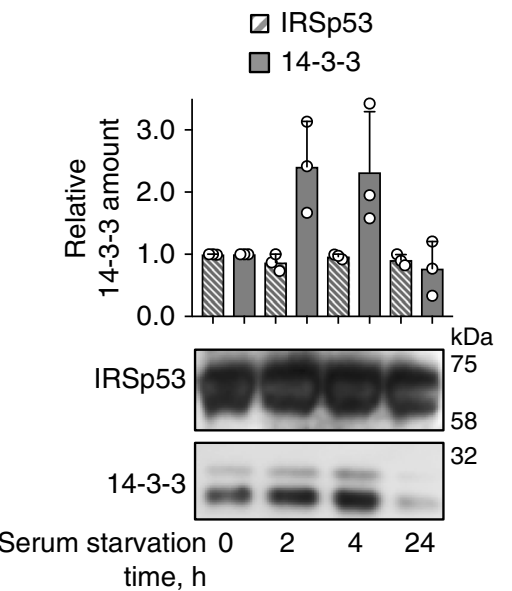

b

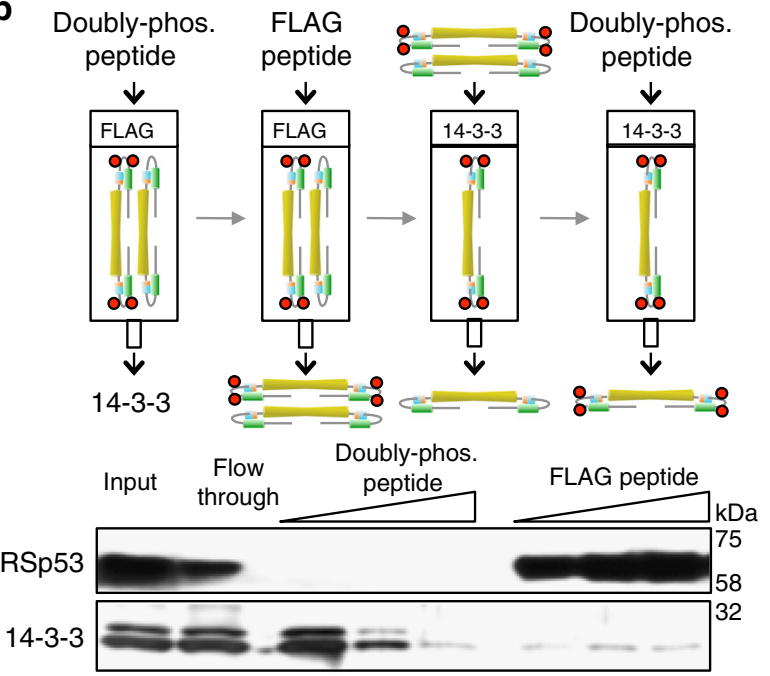

c

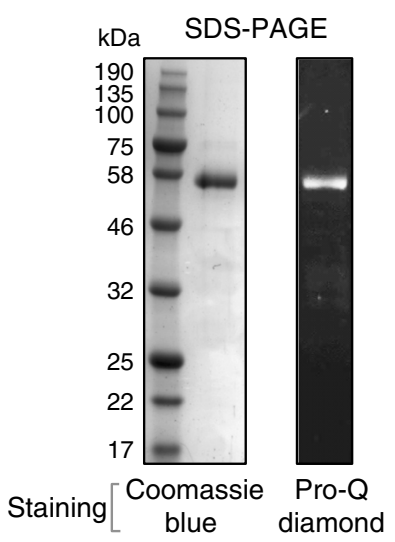

Fig. 1 Purification of natively phosphorylated and 14-3-3-binding-competent pIRSp53. a Effect of serum starvation on the relative amount of 14-3-3 (gray bars) that coimmunoprecipitates with IRSp53-FLAG (stripped bars) from HEK293T cells. Error bars are \pm s.d. from three independent experiments, shown as open circles. Abundance is reported relative to the amount of expressed IRSp53-FLAG or endogenous 14-3-3 in fed cells (first column). Note that serum starvation does not affect the expression of IRSp53-FLAG, but increases the relative amount of 14-3-3 that coimmunoprecipitates with it. An optimal starvation time of $2 \mathrm{~h}$ was established from these experiments. b Purification of phosphorylated IRSp53-FLAG from HEK293T cells. Cells were serumstarved for $2 \mathrm{~h}$ to enhance IRSp53 phosphorylation. Endogenous 14-3-3 bound to IRSp53 is competitively removed by addition of the doublyphosphorylated IRSp53 peptide Sites 2,3 (Supplementary Table 1). The 14-3-3-binding-competent fraction of IRSp53 is then isolated through consecutive affinity purification steps (FLAG-affinity and 14-3-3-affinity), eluting IRSp53-FLAG by competition with either FLAG or Sites 2,3 peptides. c Coomassie and Pro-Q Diamond-stained SDS-PAGE gels showing that the isolated fraction of IRSp53 is pure and phosphorylated 
as substrate directly phosphorylates IRSp53 at S366, and this site accounted for the largest fraction of 14-3-3 pulling down with IRSp53 35 . Par1b/MARK2 is an AMPK-related kinase ${ }^{40}$, and AMPK has been also shown to directly phosphorylate IRSp53 at S366 using a similar chemical genetics screening approach ${ }^{41,42}$. Since AMPK-related kinases are the sole to be conclusively shown to directly phosphorylate IRSp53, we used serum starvation, known to activate AMPK-dependent phosphorylation ${ }^{43}$, to enhance IRSp53 phosphorylation in HEK293T cells. As anticipated, serum starvation increased the relative amount of endogenous 14-3-3 (all isoforms included) that coimmunoprecipitates with FLAG-IRSp53, and an optimal starvation time of $2 \mathrm{~h}$ prelysis was established for future experiments (Fig. 1a). Consistent with previous findings $s^{35,41,42}$, these results indicate that AMPK (or a related kinase) phosphorylates IRSp53, and that the AMPK phosphorylation sites(s) are at least in part responsible for the binding of 14-3-335.

We developed a protocol for the purification of phosphorylated and 14-3-3-binding-competent IRSp53 (pIRSp53) from serumstarved HEK293T cells using alternating FLAG- and 14-3-3affinity steps (Fig. 1b). A FLAG peptide and a doublyphosphorylated IRSp53 synthetic peptide (Sites 2,3 peptide; see below) were used to elute IRSp53 from the affinity columns by competition. The progress of the purification was monitored by western blot analysis (Fig. 1b). SDS-PAGE analysis confirmed the purity of the purified protein, which was additionally shown to be phosphorylated using Pro-Q Diamond gel staining (Molecular Probes, Eugene, OR) (Fig. 1c).

Characterization of the 14-3-3-binding sites of pIRSp53. A survey of phosphoproteomics studies compiled in PhosphoSitePlus $^{44}$ reveals that IRSp53 is mostly phosphorylated in the flexible, S/T-rich region between the CRIB-PR and SH3 domains (Fig. 2a), which seems like an ideal location for the binding of 143-3 to inhibit interactions of the CRIB-PR with Cdc42 and the $\mathrm{SH} 3$ domain with cytoskeletal effectors. Coincidentally, two studies have identified 14-3-3-binding sites within this region, albeit different from one another: pT340/pT360 36 and S366 and to a much lesser extent a residue within the cluster ${ }^{452} \mathrm{SSST}^{455}$ located after the $\mathrm{SH} 3$ domain $^{35}$. We analyzed by mass spectrometry the highly pure, 14-3-3-binding-competent pIRSp53 preparation obtained here (Fig. 1c). Five sites were reproducibly phosphorylated in the four samples analyzed (three with $2 \mathrm{~h}$ starvation and one without starvation): S325, T340, T360, S366 and a residue within the ${ }^{452} \mathrm{SSST}^{455}$ cluster, most likely S454 (Fig. 2a, red bars, Supplementary Figure 1 and Supplementary Data 1). These sites coincide with those found with the highest frequency in phosphopoteomic studies, and include the sites previously implicated in 14-3-3 binding 35,36 .

Next, we asked which of these phosphorylation sites were implicated in 14-3-3 binding. Because the phospho-S/T-binding pockets of $14-3-3$ are $\sim 34 \AA$ apart, it can in principle bind to either two identical sites on two subunits of a dimeric target or two distinct sites on a single polypeptide, in which case the sites are commonly found 19-40 amino acids apart within intrinsically disordered regions ${ }^{45}$. Therefore, we used isothermal titration calorimetry (ITC) to test the phosphorylation sites identified here for their ability to bind Escherichia coli-expressed 14-3-30 individually and as pairs. Eight IRSp53 synthetic phosphopeptides were examined, including five 12-14-residue-long singly-phosphorylated peptides (named Site 1 to 5) and three 29-40-residue-long doubly-phosphorylated peptides (named Sites 1,$2 ; 2,3$ and 2,4) (Fig. 2a, Supplementary Table 1 and Supplementary Figure 2).
All the singly-phosphorylated peptides, except Site 1 (pS325), bound 14-3-30 with $\sim 1: 1$ stoichiometry (i.e., two peptides per 143-30 dimer) and relatively low affinities $\left(K_{\mathrm{d}}\right.$ ranging from 5.5 to $16.5 \mu \mathrm{M}$ ) (Fig. 2b, e). The doubly-phosphorylated Sites 1,2 peptide (pS325/pT340) also bound 14-3-30 with 1:1 stoichiometry and micromolar affinity (Fig. 2c, e), suggesting that it interacts with 14-3-30 only through Site 2 (pT340) thus ruling out Site 1 (pS325) as a potential 14-3-30-binding site. In contrast, the Sites 2,3 (pT340/pT360) and Sites 2,4 (pT340/pS366) peptides bound 14-3-30 with $\sim 0.5: 1$ stoichiometry (i.e., one peptide per 143-3 $\theta$ dimer) and with more than two orders of magnitude higher affinities ( $K_{\mathrm{d}}$ of $45 \mathrm{nM}$ and $67 \mathrm{nM}$, respectively) than the corresponding singly-phosphorylated peptides (Fig. 2d, e). This result suggested that these doubly phosphorylated peptides occupy the two phospho-S/T-binding pockets of 14-3-30. Of note, other targets of 14-3-3 also bind with nanomolar affinity and display similar increases in affinity for singly phosphorylated vs. doubly phosphorylated peptides ${ }^{45-51}$.

A peptide encompassing pT360 and pS366 was not analyzed, because these two sites are too close to one another in the sequence to allow for simultaneous binding to the symmetric pockets of the 14-3-3 dimer. Site 5 (pS454), which alone bound 14-3-3 $\theta$ with a $K_{\mathrm{d}}=12 \mu \mathrm{M}$ (Fig. $2 \mathrm{~b}$ ), could not be tested by ITC in combination with other sites, due to the inability to make synthetic peptides of the required length. However, a 4-alanine substitution of the ${ }^{452} \mathrm{SSST}^{455}$ cluster had no effect on the relative amount of 14-3-3 that coimmunoprecipitates with IRSp53-FLAG from cells (Fig. 2f), allowing us to rule out this cluster as a 14-3-3binding site. In contrast, single alanine substitutions of residues T340, T360, and S366 all significantly reduced 14-3-3 binding in cells, and any combination of these mutations completely abolished 14-3-3 binding (Fig. 2f). Importantly, phosphomimetic mutations of these sites cannot substitute for phosphorylation in the case of 14-3-3 binding; E. coli-expressed phosphomimetic mutants of full-length IRSp53 containing aspartic acid or glutamic acid at positions $340,360,366$, and 454 failed to bind 14-3-3 $\theta$ (Supplementary Figure 3). From the ensemble of these results we conclude that $14-3-3$ binds to three major sites in pIRSp53 (pT340, pT360, and pS366), and its binding affinity increases more than 100 folds for two pairs of sites: pT340/pT360 and pT340/pT366. The existence of two pairs of 14-3-3-binding sites in pIRSp53 reconciles the apparent discrepancy between the findings of two previous studies ${ }^{35,36}$.

Each subunit of the pIRSp53 dimer binds one 14-3-3 dimer. Both 14-3-3 and IRSp53 form constitutive dimers, as do several targets of 14-3-3, including p53, c-Raf, LRRK2, ASK1 and HSPB6. A recent crystal structure of HSPB6 in complex with 14-3-3 $\sigma^{52}$ and a SAXS study of pASK1-CD in complex with $14-3-3 \zeta^{53}$ show a single 14-3-3 dimer bound to a single phosphorylation site within one of the subunits of these dimeric targets. However, 143-3 binds to some targets through two or more phosphorylation sites, and whether 14-3-3 binds to different sites within a single subunit or to identical (or different) sites on two subunits of a dimeric target is a question that has not been formally addressed. We do this here for IRSp53, both biochemically and in cells.

In cells, a dual tag (FLAG, STREP) approach was developed for the purification of heterodimers of WT IRSp53 and a mutant in which residues T340, T360, S366, and ${ }^{452}$ SSST $^{455}$ were simultaneously mutated to alanine (M2345). Importantly, a first attempt, using two separate plasmids to co-express WT IRSp53-FLAG and M2345-STREP in HEK293T cells (Supplementary Figure 4a), failed to produce heterodimers (Supplementary Figure 4b). This approach produced only homodimers (WT/WT or M2345/ 
a
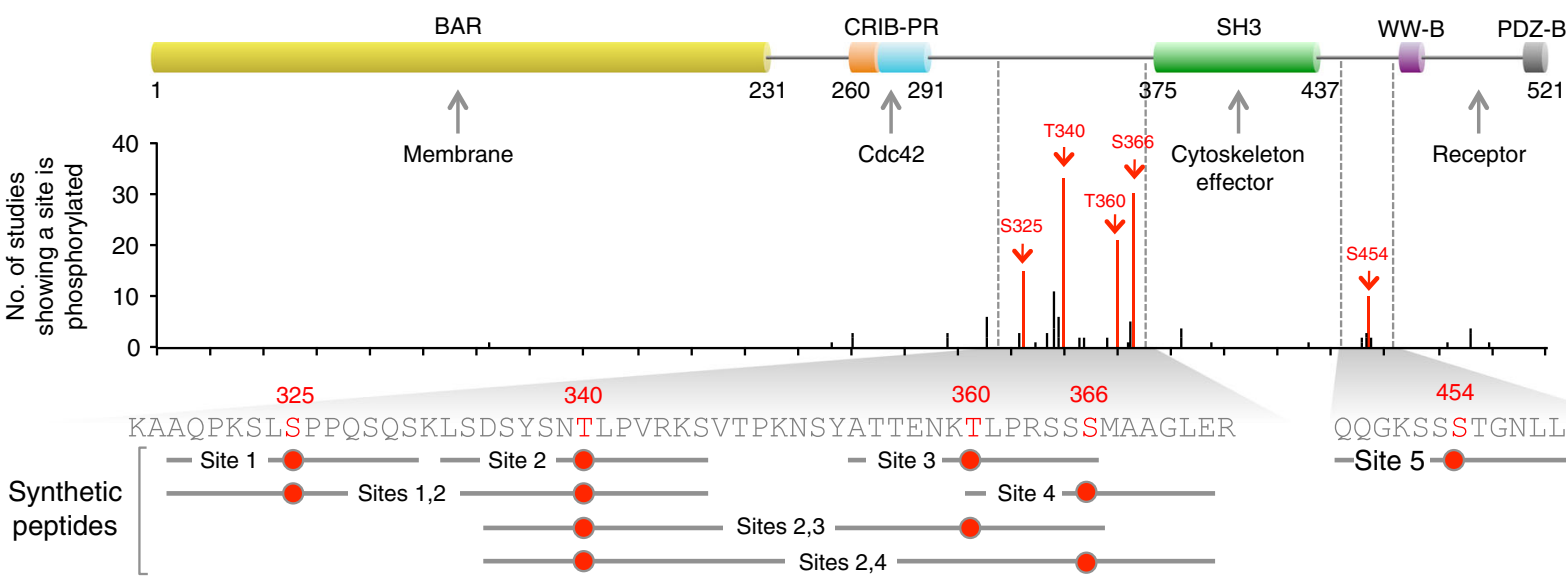

b

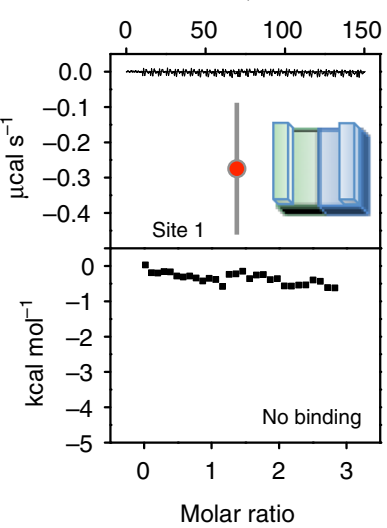

c

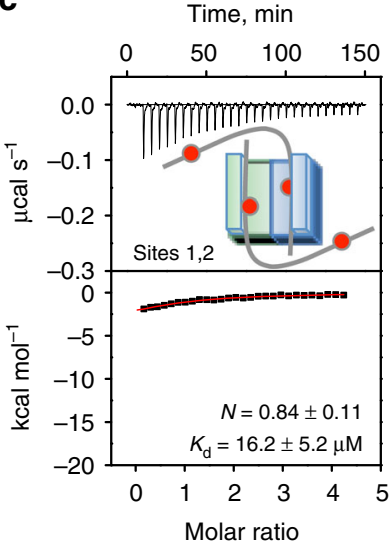

Time, $\min$

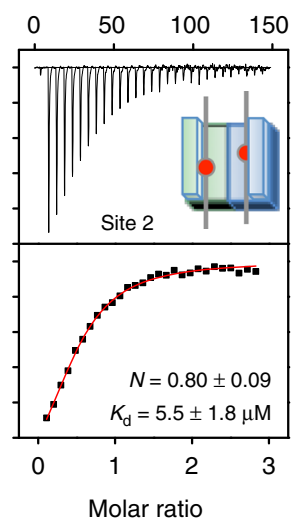

d

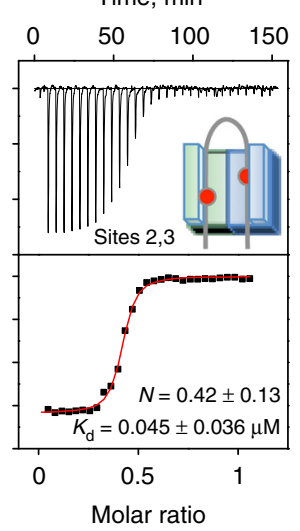

Time, $\min$
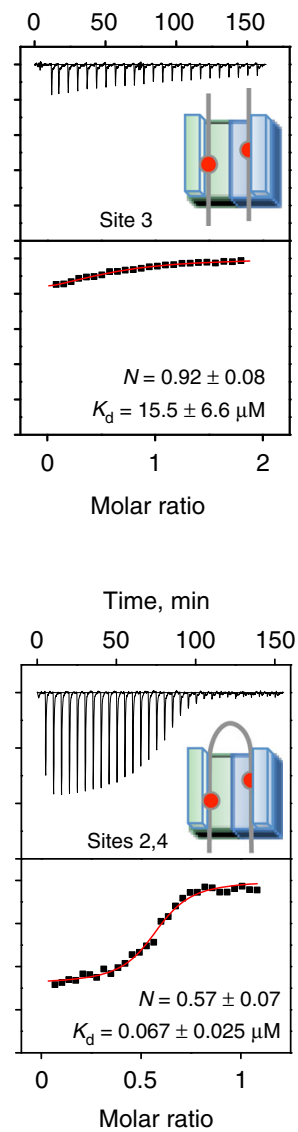

Time, $\min$
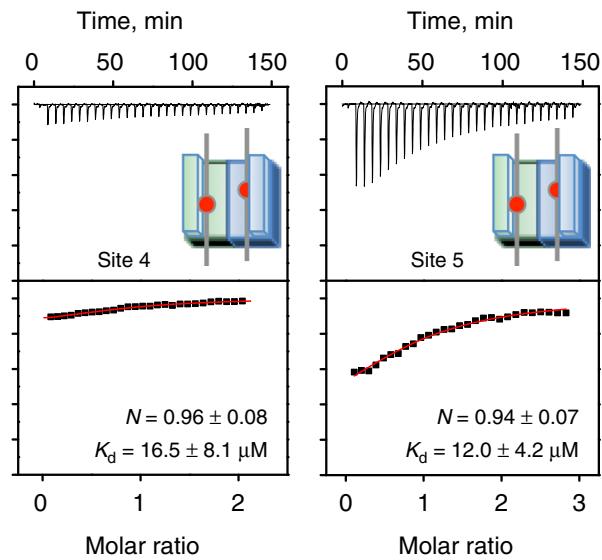

e
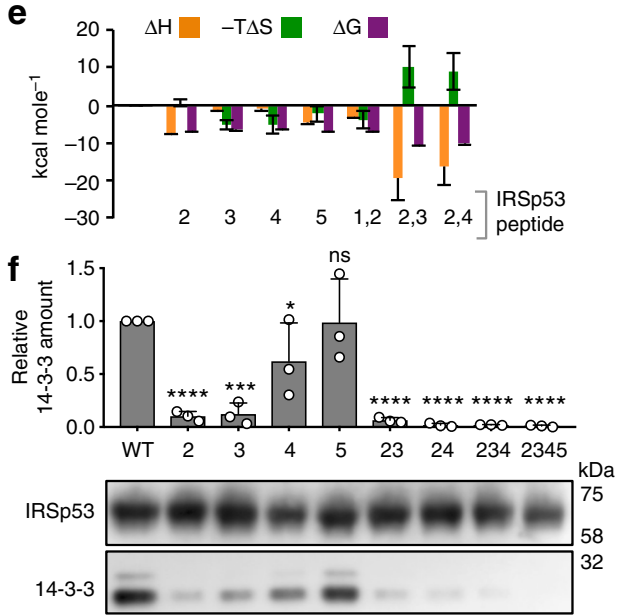

Fig. 2 Characterization of the 14-3-3-binding sites of pIRSp53. a Domain organization of IRSp53, showing the location of phosphorylation sites and the definition of synthetic phospho-peptides used in this study. In the graph, the height of each phosphorylation site represents the number of times a particular residue has been found to be phosphorylated in phosphoproteomics studies curated by PhosphoSitePlus (www.phosphosite.org). Among these, five sites (highlighted red) were consistently found to be phosphorylated in four different pIRSp53 samples analyzed here (purified as shown in Fig. 1). Note that the five sites identified here coincide with the highest frequency sites in PhosphoSitePlus. b-d ITC titrations of $100 \mu \mathrm{M}$ singly phosphorylated (Site 1 to 5) or $50 \mu \mathrm{M}$ doubly-phosphorylated (Sites 1,2; 2,3 and 2,4) peptides into $10 \mu \mathrm{M} 14-3-30$ (as indicated). ITC experiments were performed at $20^{\circ} \mathrm{C}$. Listed for each experiment are the dissociation constant $\left(K_{\mathrm{d}}\right)$ and binding stoichiometry $(N)$ derived from fitting of the binding isotherm (red line). Errors correspond to the s.d. of the fits. e Change in thermodynamic parameters $\Delta \mathrm{H}$ (orange bars), $-\mathrm{T} \Delta \mathrm{S}$ (green bars), and $\Delta \mathrm{G}$ (purple bars), derived from the fits of the ITC titrations shown in panels b-d. Errors correspond to the s.d. of the fits. f Coimmunoprecipitation of 14-3-3 with WT IRSp53-FLAG and mutants carrying $\mathrm{S} / \mathrm{T}->\mathrm{A}$ substitutions of the indicated phosphorylation sites (named according to the number of the five major phosphorylation sites shown in part a). Abundance is reported relative to the amount of endogenous 14-3-3 that coimmunoprecipitates with WT IRSp53-FLAG in serum-starved cells (first column). Error bars are \pm s.d. from three independent experiments, shown as open circles. The statistical significance of the measurements was determined using an unpaired two-sided Student's $t$-test, comparing each of the mutants to WT IRSp53 (ns, non-significant; ${ }^{\star} p<0.05 ;{ }^{\star \star \star} p<0.001$; ${ }^{\star \star \star \star} p<0.0001$ ) 
a

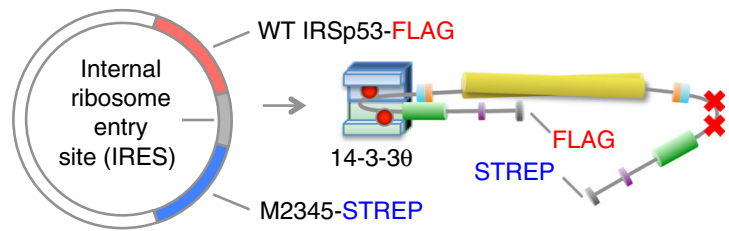

(T340A/T360A/S366A/452SSST 455 -> AAAA)

b
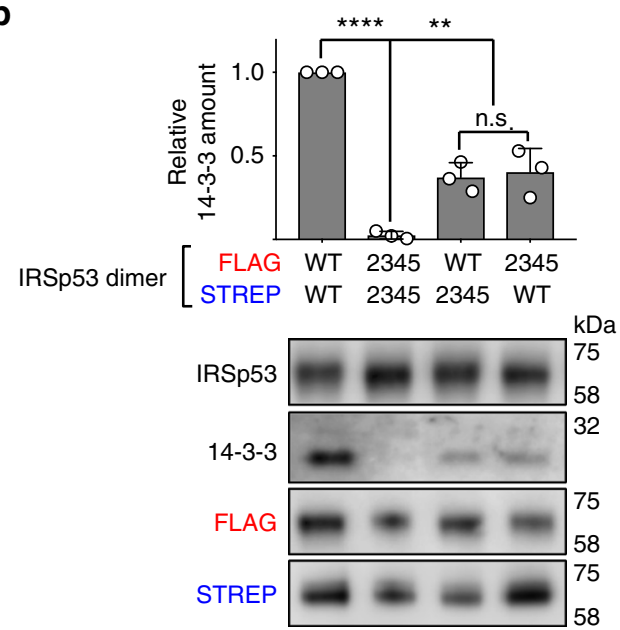

C

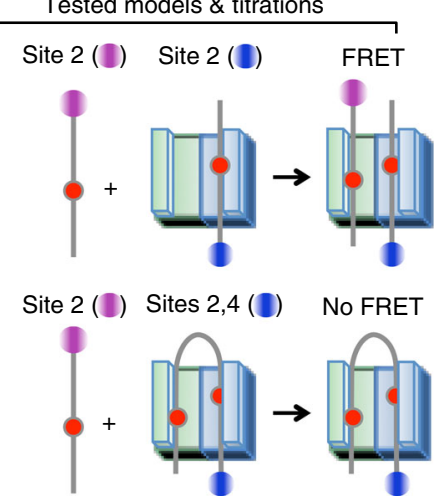

Measured FRET

- Acceptor (Alexa 568)

Donor (Alexa 488)

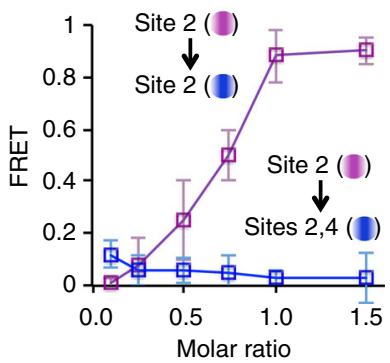

$\mathbf{d}_{\mathrm{Te}}$

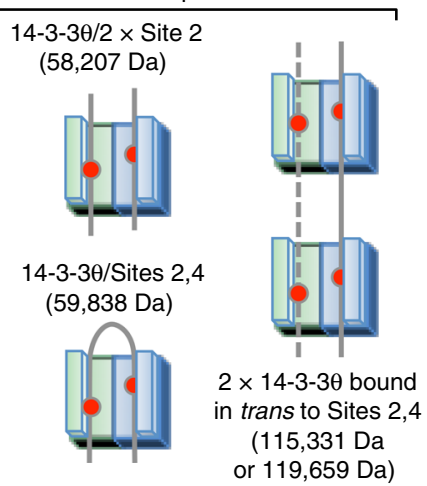

Masses measured by MALS

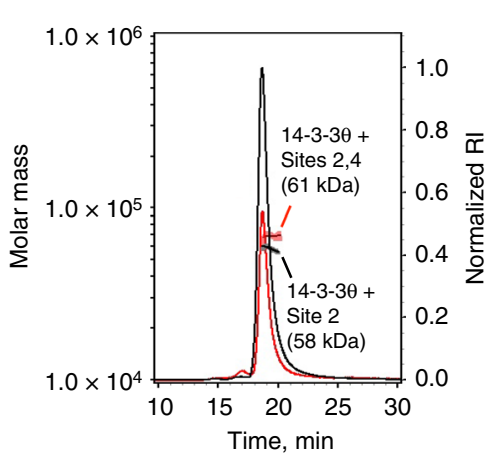

Fig. 3 Each subunit of the pIRSp53 dimer binds one 14-3-3 dimer. a Illustration of the strategy used to obtain wild-type/mutant IRSp53 heterodimers using dual affinity purification tags (FLAG and STREP) and an internal ribosomal entry site (IRES) inserted between the wild-type and mutant coding sequences of a single expression plasmid. The mutant M2345 carries seven S/T- > A substitutions (T340A/T360A/S366A/452SSST455 - > AAAA). b Coimmunoprecipitation of 14-3-3 with homo- and heterodimers of wild-type and mutant IRSp53. Two different heterodimers were tested, where the mutant subunit either preceded or followed the IRES, which also changed the purification tag. Abundance is reported relative to the amount of endogenous 14-3-3 that coimmunoprecipitates with WT-IRSp53-FLAG/WT-IRSp53-STREP. Error bars are \pm s.d. from three independent experiments, shown as open circles. The statistical significance of the measurements was determined using an unpaired two-sided Student's $t$-test, comparing each of the samples to wild-type IRSp53 (ns, non-significant; ${ }^{* \star} p<0.01$; ${ }^{* \star \star} p<0.0001$ ). c FRET assay in which acceptor (Alexa 568)-labeled Site 2 peptide was titrated into 14-330 half-saturated (1:0.5 molar ratio) with donor (Alexa 488)-labeled Site 2 (magenta squares) or Sites 2,4 peptides (blue squares). FRET is only observed when the acceptor-labeled peptide binds to one of the pockets of the 14-3-30 dimer while the other pocket is occupied by donor-labeled peptide (as depicted on the left). Error bars are \pm s.d. from three independent experiments. d Determination of the masses of complexes of 14-3-30 with Site 2 (black line) or Sites 2,4 (red line) peptides using SEC-MALS. The tested interaction models and corresponding masses are shown on the left

M2345), strongly suggesting that IRSp53 dimerization occurs during translation and is irreversible. In contrast, WT/M2345 heterodimers were successfully expressed from a single vector, coding a single transcription product. For this, we inserted an internal ribosomal entry site (IRES) between the WT and M2345 coding sequences, allowing for the simultaneous translation of two open reading frames (Fig. 3a). When both subunits were mutated, 14-3-3 did not coimmunoprecipitate with M2345/ M2345, whereas $~ 50 \%$ of the amount that coimmunoprecipitates with WT/WT IRSp53 was observed when one of the dimer subunits was mutated (WT/M2345 or M2345/WT), independent of which affinity tag was attached to the mutant subunit or whether the mutant subunit preceded or followed the IRES (Fig. 3b). These results demonstrate that 14-3-3 binds to two different phosphorylation sites on a single subunit of the pIRSp53 dimer in cells.

To further validate the interaction of 14-3-3 with pairs of sites within a single subunit of the pIRSp53 dimer, a FRET competition assay was developed to monitor the binding of acceptor-labeled Site 2 peptide to half-saturated
(1:0.5 stoichiometry) complexes of 14-3-30 with either donorlabeled Site 2 or Sites 2,4 peptides (Fig. 3c). The titration into the half-saturated 14-3-30/Site 2 complex produced a large FRET increase, indicating that the acceptor-labeled Site 2 peptide bound to the vacant pocket of the 14-3-30 dimer, within FRET distance from the donor-labeled Site 2 peptide (pre-bound to the other pocket of 14-3-30). In contrast, the titration of acceptor-labeled Site 2 peptide into the 14-3-30/Sites 2,4 complex did not produce a FRET change, suggesting that both pockets of $14-3-3 \theta$ were occupied by the doubly phosphorylated peptide, and that the singly phosphorylated peptide cannot outcompete the doubly phosphorylated peptide from either pocket.

A doubly-phosphorylated peptide could in principle bind to two 14-3-30 dimers in trans, a possibility tested here using multiangle light scattering to measure the masses of saturated complexes of 14-3-30 with singly- and doubly-phosphorylated peptides (Fig. 3d). The experimentally determined mass of the 143-30/Site 2 complex was $58,000 \mathrm{Da}$, consistent with a 1:1 complex (theoretical mass: $58,207 \mathrm{Da}$ ), whereas that of 14-3-30/Sites 2,4 was $61,000 \mathrm{Da}$, corresponding to a 1:0.5 complex (theoretical 
mass: $59,838 \mathrm{Da}$ ). These masses rule out the formation of 14-3-30 complexes in trans with the doubly-phosphorylated Sites 2,4 peptide, with theoretical masses of 115,331 Da (one peptide bound in trans to two $14-3-3 \theta$ dimers) or $119,659 \mathrm{Da}$ (two peptides bound in trans to two 14-3-30 dimers). Together, these results suggest that 14-3-3 binds to two pairs of sites (pT340/pT360 or pT340/pS366) within a single subunit of the IRSp53 dimer.

\section{Structural basis for the interaction of 14-3-3 with pIRSp53.} Three factors make the structural characterization of complexes of 14-3-3 with doubly-phosphorylated peptides challenging: (a) the low-complexity regions between phosphorylation sites are usually disordered in the structures of 14-3-3 complexes, (b) 143-3 binds to phospho-S/T sites that are related in sequence and thus difficult to tell apart in electron density maps, and (c) doubly-phosphorylated peptides can in principle bind with two opposite N- to C-terminal orientations, such that each of the 143-3 pockets could in principle contain a mixture of the two phosphorylation sites present in the peptide. Therefore, to study the binding of $14-3-3 \theta$ to the pT340/pT360 and pT340/pS366 pairs, we determined five crystal structures, allowing for direct comparisons of the structures of singly- vs. doublyphosphorylated complexes (Fig. 4 and Supplementary Figure 5). In the structures, ranging in resolution from 2.0 to $2.9 \AA$ (Supplementary Table 2), the electron density maps revealed the phosphorylated residue and 3 to 5 residues $\mathrm{N}$ - and C-terminal to it, whereas the ends of the peptides and the flexible linkers between phosphorylation sites in the doubly-phosphorylated peptides were unresolved. In all the structures, the phosphate group on the phosphorylated side chain engages a cluster of basic residues in 14-3-30, consisting of K49, R56, and R127, and additionally forms a hydrogen bond with the hydroxyl group of Y128. For the singly-phosphorylated peptides, the electron density is symmetric for the two pockets of 14-3-30 (Supplementary Figure 6), even when two-fold symmetry was not imposed during refinement. Importantly, the structures of the singlyphosphorylated peptides display distinctive features that are recognizable in those of the doubly-phosphorylated peptides, for which the electron density is asymmetric in the two pockets of 143-3 $\theta$ (Fig. 4 and Supplementary Figure 7). Specifically, the Site 2 and Site 3 peptides contain a proline residue at the second position after the phosphorylation site, producing a characteristic $90^{\circ}$ bend of the polypeptide chain, which as a result exits the 143-3-binding groove, thus leaving a significant portion of the groove unoccupied (Supplementary Figure 5a, b and Supplementary Figure $6 \mathrm{a}, \mathrm{b})$. In contrast, the Site 4 peptide has an alanine residue at the second position after the phosphorylation site (pS366), and follows a relatively straight path along the groove of 14-3-30 (Supplementary Figure $5 \mathrm{c}$ and Supplementary Figure 6c). Although the sequences surrounding pT340 and pT360 are closely related to each other, they are distinguishable by the presence of two large side chains in the Site 1 peptide, Y337, and R344, occupied by smaller side chains in the Site 2 peptide, E357, and S365, respectively (Supplementary Figure 5a, b). These distinctive features of the singly-phosphorylated peptides are clearly discernable in the structures of the doublyphosphorylated peptides (Fig. 4a, b), in which each pocket of 143-3 $\theta$ binds a different site in an asymmetric manner. We note, however, that the structure of the pT340/pT360 pair contains four complexes in the P1 unit cell, and only one of these complexes has a unique $\mathrm{N}$ - to C-terminal orientation of the doubly phosphorylated peptide, whereas the other three complexes appear to have a mixture of orientations. Together, the biochemical (Figs. 2 and 3) and structural (Fig. 4) data allow us to conclude that 14-3-
3 binds with high affinity to two pairs of sites in pIRSp53, pT340/ pT360, and pT340/pS366.

A FRET-sensor assay shows inhibition of pIRSp53 by 14-3-3. We then asked whether the binding of 14-3-3 to pIRSp53 regulates its activity by stabilizing the closed, autoinhibited conformation in which an intramolecular interaction between the CRIB-PR and SH3 domains blocks access to these two domains ${ }^{10}$. To test this idea, a FRET-sensor assay was developed to monitor conformational changes in pIRSp53 as a function of its interaction with binding partners. This approach builds upon our previous report of a FRET-sensor using E. coli-expressed IRSp53 $3^{10}$, but we now use the purification protocol described here (Fig. 1) to obtain a FRET sensor natively phosphorylated in mammalian cells $\left(\mathrm{pIRSp} 53_{\mathrm{FS}}\right.$ ). The protein used in the FRET-sensor assay was labeled with the donor/acceptor pair fluorescein/rhodamine at two positions: endogenous C230 within the BAR domain (the only label-accessible Cys residue in IRSp53) and C519 introduced by mutagenesis near the C-terminus (S519 in WT IRSp53). Conformational changes in $\mathrm{pIRSp} 53_{\mathrm{FS}}$ were monitored as the change in energy transfer resulting from the binding of several inputs: 14-3-30, Cdc42 and the cytoskeletal effector Eps8 (Fig. 5a). All the proteins used in these experiments were purified to homogeneity (Supplementary Figure 8).

The titration of GMPPNP-Cdc42 (constitutively active mutant $\mathrm{G} 12 \mathrm{~V}$ ) into pIRSp53 $\mathrm{FS}$ induced a transition toward an open, active conformation, characterized by an increase in the donor fluorescence (i.e., a low FRET state) (Fig. 5b). The subsequent titration of 14-3-3 $\theta$ into this complex reduced the donor fluorescence (high FRET state), consistent with a reversal of the conformational change induced by $\mathrm{Cdc} 42$ and a transition of pIRSp53 toward a closed, inactive conformation. When the order of the titrations was inverted, 14-3-3 $\theta$ triggered a transition of pIRSp $53_{\mathrm{FS}}$ toward a closed conformation, which remained mostly unchanged with the subsequent titration of Cdc42 (Fig. 5c). The reason for the small increase in energy transfer upon titration with Cdc42 is unclear, but could indicate non-activatory binding of Cdc42 to the IRSp53-14-3-3 complex. These results indicate that Cdc42 cannot competitively activate pIRSp53 once it is bound to 14-3-3.

Since, the binding of cytoskeletal effectors such as Eps8 to the $\mathrm{SH} 3$ domain also activates IRSp53 ${ }^{10}$, we performed back-to-back titrations of $14-3-3 \theta$ and Eps8 into pIRSp53 ${ }_{\mathrm{FS}}$, using Eps8 purified from mammalian cells (Supplementary Figure 8). The binding of Eps8 to pIRSp53 $\mathrm{FS}$ increased the donor fluorescence in a cooperative manner (Fig. 5d), consistent with a transition of pIRSp53 toward an open, active state. As we have shown before ${ }^{10}$, the Eps8/pIRSp53 complex has 2:2 stoichiometry. However, the subsequent titration of 14-3-30 into this complex did not revert the conformational change produced by Eps8. On the contrary, the donor fluorescence increased linearly, albeit only by $\sim 15 \%$ with a 4-fold molar excess of 14-3-30 to Eps8 (Fig. 5d). This effect is possibly explained by the inability of $14-3-3 \theta$ to displace Eps8 from its complex with pIRSp53, as also suggested by the observation that FLAG-tagged Eps8 pulls down both pIRSp53 and $14-3-3 \theta$ (Fig. 5f). We thus conclude that the binding of 14-3$3 \theta$ to Eps8/pIRSp53 produces a low-FRET ternary complex, whose conformation is distinct from those of the active Eps8/ pIRSp53 and the inhibited 14-3-30/pIRSp53 complexes, and which we cannot reliable classify as either open or closed. The ability of $14-3-3 \theta$ to competitively revert the conformational change induced by $\mathrm{Cdc} 42$ but not Eps 8 is consistent with the relative binding affinities of these three proteins for pIRSp53; from fitting of the titrations the observed $K_{\mathrm{d}}\left(K_{\mathrm{obs}}\right)$ of the 
a 340 360 ${ }^{334}$ SDSYSNTLPVRKSVTPKNSYATTENKTLPRSS S ${ }^{366}$
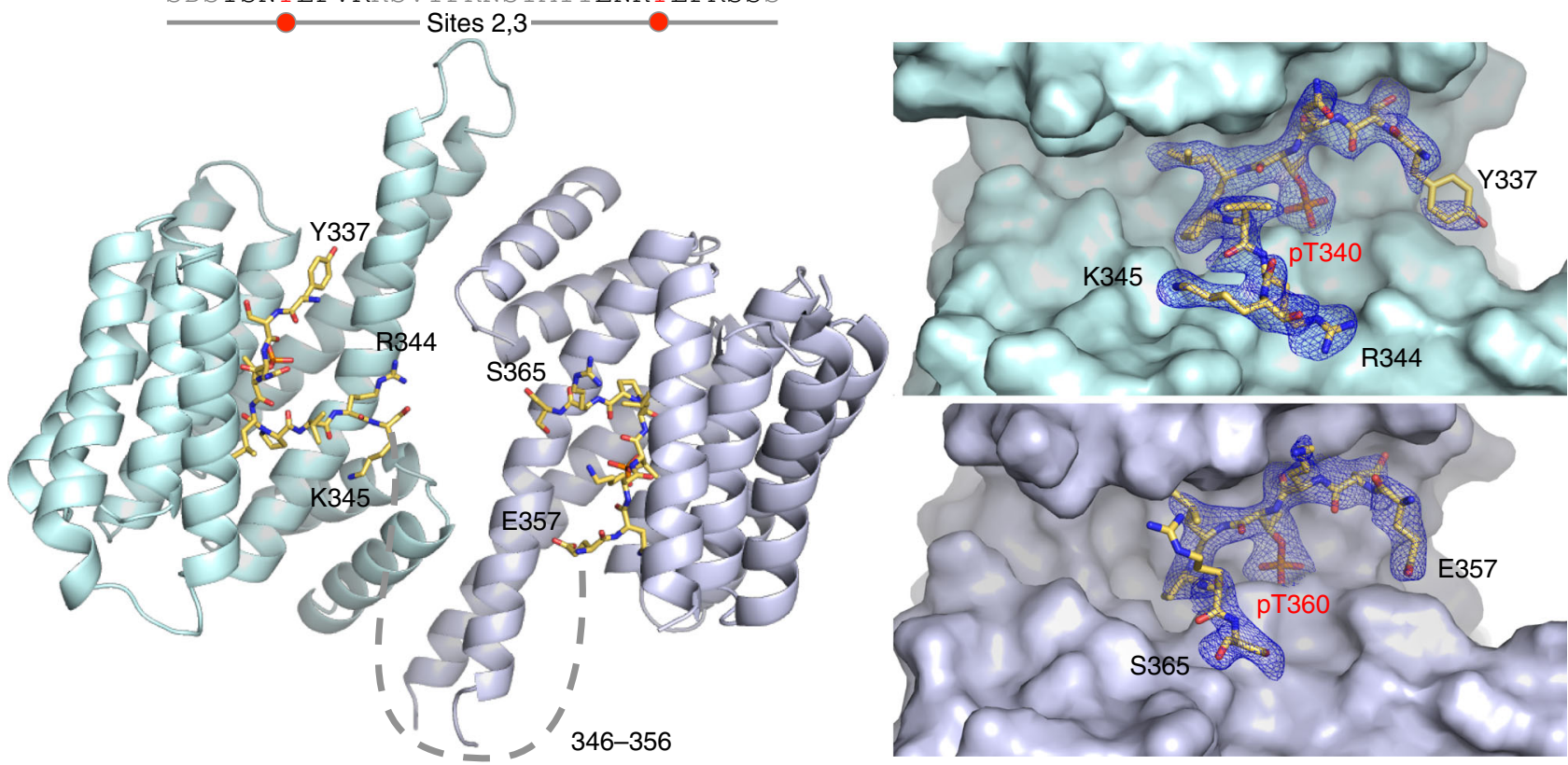

b 340
${ }^{334}$ SDSYSNTLPVRKSVTPKNSYYTTENKTLPRSSSMAAGLER
373
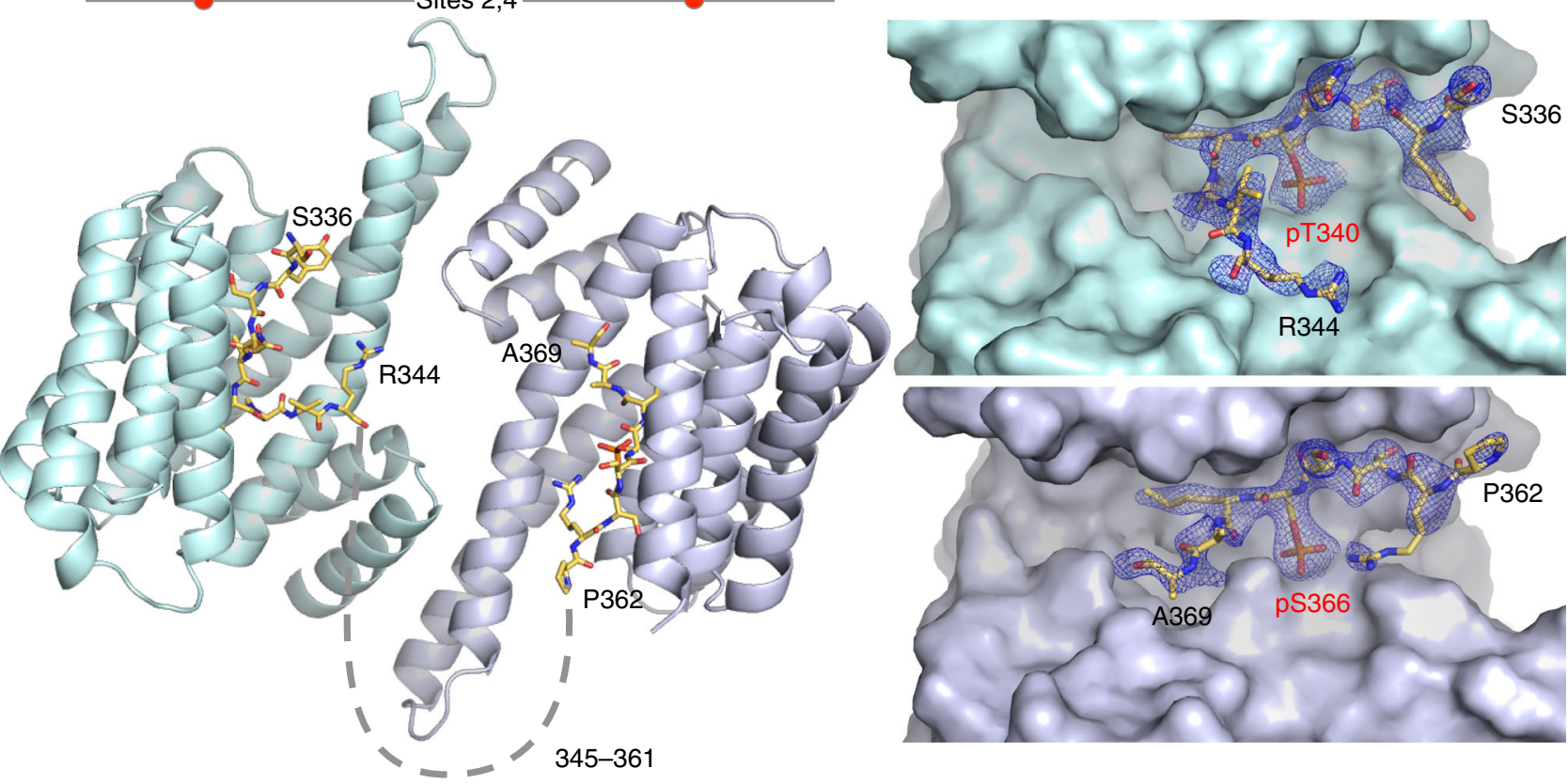

Fig. 4 Structures of complexes of 14-3-30 with doubly-phosphorylated IRSp53 peptides. a, b Complexes of 14-3-30 with the Sites 2,3 and Sites 2,4 peptides, showing the overall structure (left) and close-ups of the binding pockets of $14-3-3 \theta$ and corresponding $2 F_{\mathrm{o}}-F_{\mathrm{c}}$ electron density maps contoured at $1 \sigma$ (right). In the sequence diagrams shown, the portions of the peptides observed in the structures are highlighted black and the phosphorylation sites are highlighted red, whereas unresolved amino acids are shown in gray. Dashed lines indicate the path between the observed portions of the bound peptides in the structures. Note that the sequences around the two phosphorylation sites of each peptide are different enough to be clearly distinguishable in the electron density maps in the separate pockets of 14-3-30 (see also Supplementary Figure 7), as confirmed by cross-comparisons with the structures of the corresponding singly-phosphorylated peptides (Supplementary Figures 5 and 6)

interactions of pIRSp53 with Cdc42, Eps8, and 14-3-30 were estimated at $19.1 \mu \mathrm{M}, 0.042 \mu \mathrm{M}$, and $0.079 \mu \mathrm{M}$, respectively. We then inverted the order of the titrations; $14-3-3 \theta$ induced the expected transition of pIRSp53 toward a closed, inactive state, and the subsequent titration of Eps8 into this complex did not change the donor fluorescence (Fig. 5e), suggesting that even a high-affinity cytoskeletal effector such as Eps8 cannot competitively activate pIRSp53 once it is bound to 14-3-3.

To test whether the effect of $14-3-3 \theta$ on the conformation of pIRSp53 depends on binding to the two pairs of phosphorylation sites identified above, we expressed in serum-starved HEK293T cells a modified version of the FRET sensor (pM234 ${ }_{\mathrm{FS}}$ ) 
a
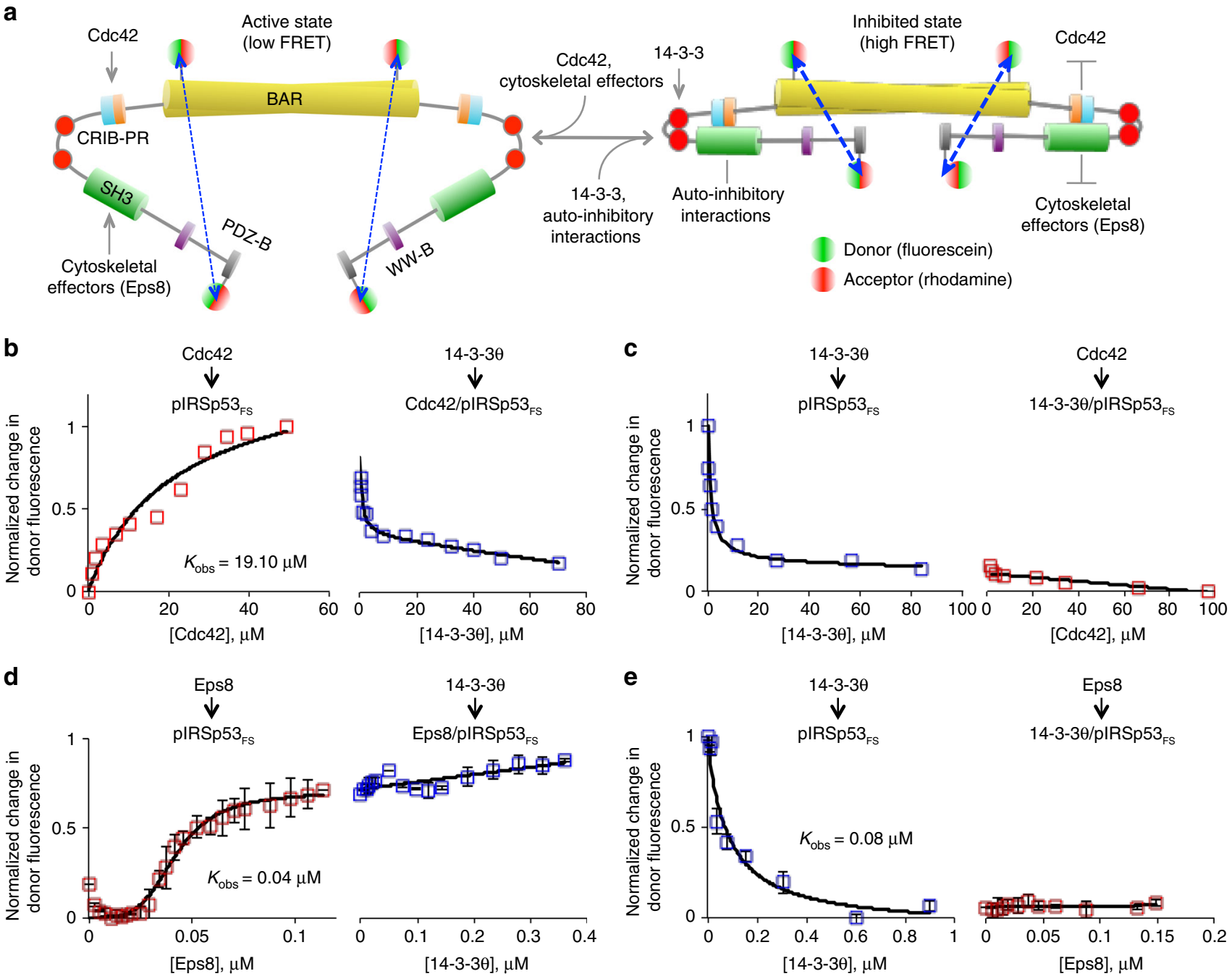

$\mathbf{f}$
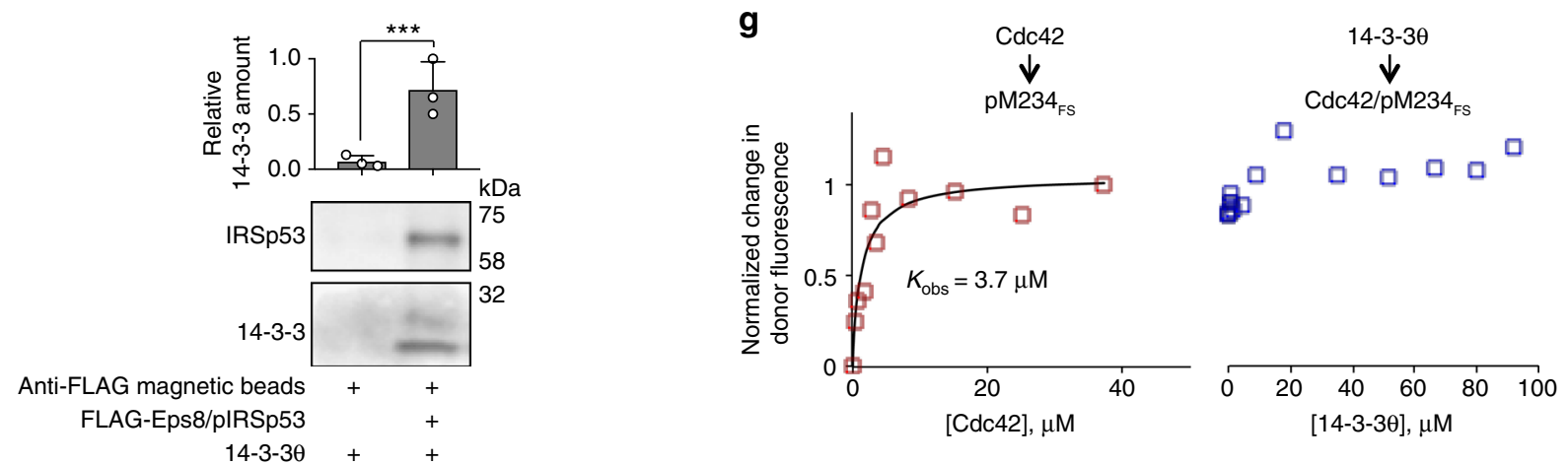

Fig. 5 A FRET-sensor assay shows inhibition of pIRSp53 by 14-3-3. a FRET sensor assay designed to report on conformational changes occurring in pIRSp53 as a function of interactions with binding partners. Phosphorylated IRSp53 (mutant S519C) was expressed and purified as shown in Fig. 1 and labeled at C230 (within the BAR domain) and C519 (near the C-terminus) with the donor/acceptor pair fluorescein/rhodamine (pIRSp53 $3_{\mathrm{FS}}$ ). The same batch of probes and donor/acceptor ratio were used in all labeling reactions. Conformational changes in pIRSp53 $3_{\mathrm{FS}}$ are detected based on the amount of FRET between the fluorescence probes at these two distant positions. b, c FRET titration of $2 \mu \mathrm{M}$ GNPPNP-Cdc42 (G12V mutant) into $0.2 \mu \mathrm{M}$ pIRSp53 FS (red squares), followed by the titration of $0.2 \mu \mathrm{M} 14-3-3 \theta$ (blue squares) and reverse-order titration. d, e FRET titration of $0.2 \mu \mathrm{M} \mathrm{Eps} 8$ into $0.05 \mu \mathrm{M}$ pIRSp53 (red squares), followed by the titration of $4 \mu \mathrm{M} 14-3-30$ (blue squares) and reverse-order titrations. Pairs of titrations were normalized to the total change in FRET. Each titration was fit to a non-cooperative binding function (black lines), except that of Eps8 into pIRSp53 Fs, which was fit to a cooperative binding function with non-specific binding (see Methods). $\mathbf{f}$ Relative abundance of 14-3-3 pulling down with anti-Flag magnetic beads in the presence or the absence of the FLAG-Eps8/pIRSp53 complex. Error bars are \pm s.d. from three independent experiments, shown as open circles. The statistical significance of the measurements was determined using an unpaired two-sided Student's $t$-test $\left({ }^{\star \star} p<0.001\right)$. $\mathbf{g}$ FRET titration of $2 \mu \mathrm{M}$ GNPPNP-Cdc42 into $0.2 \mu \mathrm{M}$ mutant M234 Fs (red squares), followed by the titration of $0.2 \mu \mathrm{M} 14-3-30$ (blue squares) 
a

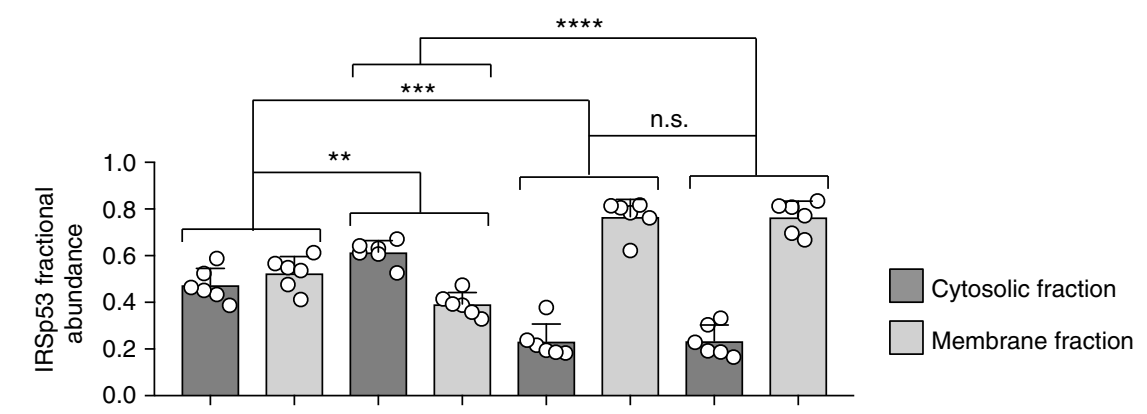

Starvation time (h)

0

2

0

2

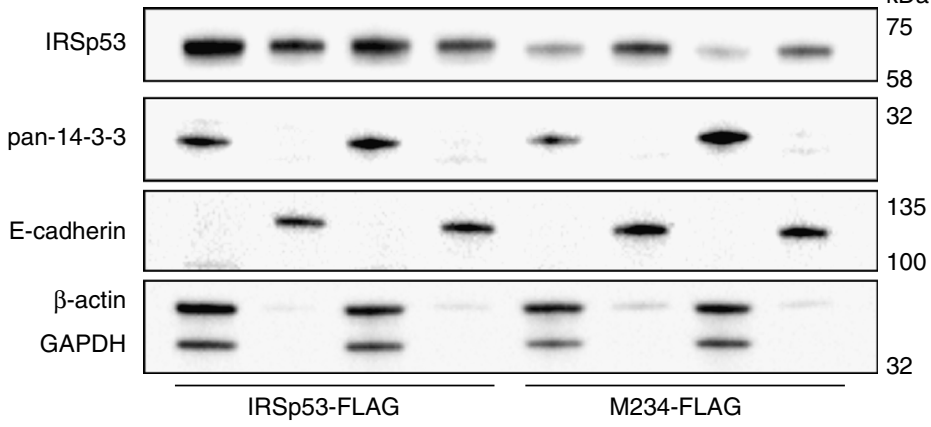

b

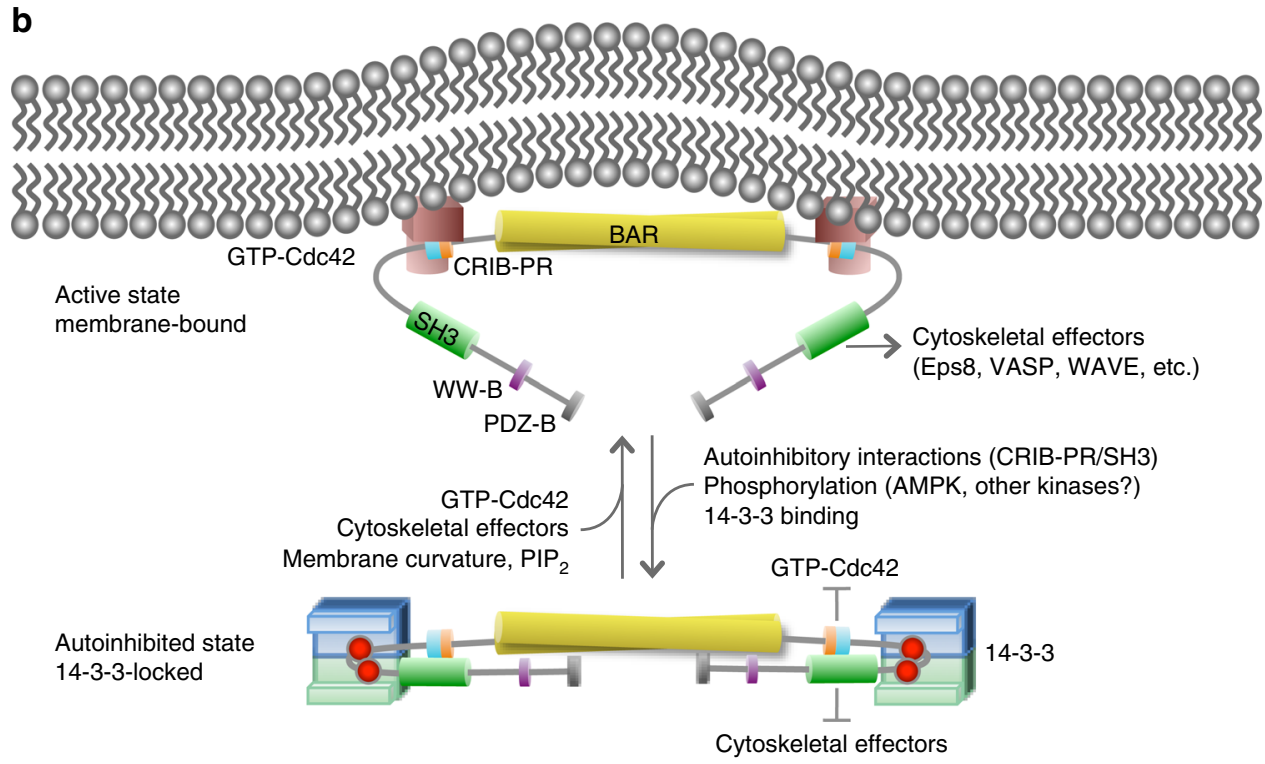

Fig. 6 Mechanism of IRSp53 regulation by 14-3-3. a 14-3-3 inhibits membrane binding of pIRSp53. The relative abundances of WT IRSp53-FLAG and mutant M234-FLAG in the cytosolic (black bars) and membrane fractions (gray bars) of fed and serum-starved HEK293T cells, were determined by weighting the abundance of IRSp53 or M234 against the combined abundance of GAPDH and $\beta$-actin, for the cytosolic fraction, or E-cadherin, for the membrane fraction. Error bars are \pm s.d. from three different transfections and two experiments per transfection (shown as open circles). The statistical significance of the measurements was determined using an unpaired two-sided Student's $t$-test (n.s., non-significant; ${ }^{\star \star} p<0.01$; ${ }^{\star \star \star} p<0.001$; ${ }^{\star \star \star \star} p<$ 0.0001). b Model of IRSp53 activation and phosphorylation-dependent inhibition by 14-3-3. IRSp53 is activated by the binding of Cdc42 to the CRIB-PR domain and/or cytoskeletal effectors to the SH3 domain. Activation exposes the I-BAR domain for binding to the plasma membrane. Phosphorylation of IRSp53 and subsequent binding of 14-3-3 to phosphorylation sites located between the CRIB-PR and SH3 domains stabilizes the autoinhibited conformation and inhibits the binding of $\mathrm{Cdc} 42$ and downstream cytoskeletal effectors

in which these sites were simultaneously mutated to alanine (T340A/T360A/S366A). As shown above (Fig. 2f), this mutant fails to coimmunoprecipitate with 14-3-3 from cells. The titration of Cdc42 into pM234 $4_{\mathrm{FS}}$ induced the anticipated transition to an open conformation, but contrary to pIRSp $53_{\mathrm{FS}}$ the subsequent titration of $14-3-3 \theta$ did not revert this conformational change (compare Fig. 5b, g). Together, these results suggest that 14-3-3 locks pIRSp53 in a closed, autoinhibited conformation, blocking the interactions of $\mathrm{Cdc} 42$ and cytoskeletal effectors with the CRIB-PR and SH3 domains.

14-3-3 inhibits pIRSp53 binding to cellular membranes. We then asked whether the binding of 14-3-3 inhibits the interaction of pIRSp53 with membranes in cells. To this end, we separated the cytosolic and membrane fractions of fed and serum-starved 
HEK293T cells expressing either WT IRSp53-FLAG or the mutant M234-FLAG (unable to bind 14-3-3), and determined the relative abundance of the two IRSp53 constructs in the two cellular fractions. In fed cells, wild-type IRSp53-FLAG was rather evenly distributed between the cytosolic and membrane fractions, whereas M234-FLAG was considerably more abundant in the membrane fraction (Fig. 6a). Serum starvation, a condition that increases the amount of 14-3-3 that coimmunoprecipitates with FLAG-IRSp53 (Fig. 1a), reduced the amount of WT IRSp53FLAG bound to membranes by $\sim 13 \%$ compared to fed cells, but had no significant effect on the distribution of M234-FLAG between cytosolic and membrane fractions. We thus conclude that 14-3-3 not only inhibits the binding of pIRSp53 to Cdc42 and cytoskeletal effectors, but also interferes with its interaction with cellular membranes.

\section{Discussion}

Membrane remodeling during processes such as tissue development and cancer cell migration depends on the interplay between membrane-associated proteins and the actin cytoskeleton. The I-BAR domain protein IRSp53 is a key player in these processes, acting as a hub that integrates signaling cues from Rho-GTPases and phosphorylation pathways to initiate cytoskeleton and membrane remodeling events ${ }^{2,7}$. 14-3-3 proteins, on the other hand, are a conserved family of phospho-S/T adaptors, known primarily for their roles in the control of cell cycle progression and apoptosis ${ }^{54}$ and the regulation of cell metabolism ${ }^{55}$. Yet, 14-3-3 has been also implicated in interactions with a growing number of actin cytoskeletal proteins $35,36,39,56-59$, and it is known to impact both membrane morphology and actin cytoskeleton assembly ${ }^{56,57}$, although the mechanism is not well understood. This study provides a potential mechanism by linking $14-3-3$ to the regulation of pIRSp53.

Using a combination of structural, biochemical, and biophysical approaches, this study supports a mechanism whereby phosphorylation-dependent inhibition of pIRSp53 by 14-3-3 counters activation by $\mathrm{Cdc} 42$ and downstream cytoskeletal effectors and binding to cellular membranes (Fig. 6b). The major findings in support of this conclusion are: (a) a combination of phosphoproteomics, pull-down assays, ITC, and competitive binding analysis by FRET and light scattering of doublyphosphorylated vs. singly-phosphorylated IRSp53 peptides demonstrates that 14-3-3 binds to two pairs of sites in the linker between the CRIB-PR and SH3 domains of pIRSp53 (pT340/ pT360 or pT340/pS366), (b) analysis of an IRSp53 WT/M2345 heterodimer in which one subunit of the dimer is nonphosphorylatable shows that 14-3-3 binds to pairs of sites within a single subunit of the IRSp53 dimer, (c) the interaction of 14-3-30 with the phosphorylation sites of PIRSp53 was visualized in five crystal structures of singly- and doubly-phosphorylated IRSp53 peptides bound to 14-3-30, (d) a FRET sensor assay based on natively phosphorylated IRSp53 purified from mammalian cells shows that 14-3-3 locks pIRSp53 in a closed, autoinhibited conformation, whereby access of Cdc42 to the CRIB-PR and cytoskeletal effectors to the SH3 domain are both inhibited, (e) membrane fractionation shows greater association of mutant M234 with cellular membranes than WT IRSp53, and partial depletion of WT IRSp53 but not M234 from membranes upon serum starvation.

Some of the experimental approaches developed here may find general applicability in the study of other 14-3-3 interactions, and in particular the FRET sensor assay. Indeed, the binding of 14-3-3 often results in a conformational change that either inhibits or unleashes a new function in the target protein by masking or exposing functional domains ${ }^{60,61}$. In such cases, it is essential to understand how the binding of 14-3-3 affects the structure and interactions of its targets, for which FRET sensors provide a simple and broadly viable tool. FRET sensors may also be used to identify novel modulators of 14-3-3 interactions, which may have potential therapeutic applications $^{62}$.

There are several isoforms of IRSp53, differing primarily at the C-terminus. In this study we focused on isoform-4, which is exclusively expressed in brain, and has a PDZ-binding site at the C-terminus ${ }^{63}$. The 14-3-3-binding sites identified here (pT340, pT360, and pS366) are conserved among all the isoforms, and among different species, suggesting that the mechanism of 14-3-3 regulation described here is conserved. In the future, it would be interesting to explore the ability of 14-3-3 to regulate other members of the I-BAR family, which are generally related to IRSp53 and often linked to cytoskeleton remodeling. Algorithms that predict potential 14-3-3-binding sites could help guide such studies. In this regard, we note that among several prediction servers tested, 14-3-3-Pred, which combines three different classifiers to predict 14-3-3-binding sites ${ }^{64}$, showed the best performance. It identified five potential 14-3-3-binding sites within the region between the CRIB-PR and SH3 domains of IRSp53 (residues 292-374): S303, T340, T348, T360, and S366. This prediction includes the three major sites identified here: T340 and T360 (predicted by two classifiers) and S366 (predicted by three classifiers).

Because the 14-3-3 dimer contains two phospho-S/T-binding pockets, it could in principle bind to two ligands simultaneously, and function as a protein-protein adaptor. Yet, such mode of binding is rare, as the majority of 14-3-3 ligands contain two or more 14-3-3-binding sites that simultaneously engage the two pockets of the 14-3-3 dimer ${ }^{45,46,61}$. Therefore, our finding here that 14-3-3 inhibits pIRSp53 by binding to two different pairs of phosphorylation sites is far from unique, and instead suggests the existence of alternate routes of IRSp53 regulation, likely involving different kinases. The two pairs of 14-3-3-binding sites in pIRSp53 have one site in common, pT340, suggesting that pT360 and pS366 act as secondary sites in these pairs. This mode of action is consistent with the gatekeeper model, according to which phosphorylation of a first, high-affinity site leads to the initial recruitment of 14-3-3 to a multi-site ligand, followed by phosphorylation and binding of the second pocket of 14-3-3 to a weaker secondary site, which in turn triggers a conformational change in the target protein ${ }^{60}$. Coincidentally, 14-3-3 binds with 3-fold higher affinity to pT340 than to pT360 or pS366, whereas its affinity for the two pairs of sites is more than 100 fold higher than for individual sites.

The identity of the kinases implicated in IRSp53 phosphorylation, and specifically at 14-3-3-binding sites, is mostly unknown. The exception is AMPK and AMPK-related kinases, which studies using chemical genetics screening have conclusively implicated in direct phosphorylation of IRSp53 at S366 $35,41,42$. Furthermore, one of these studies linked the phosphorylation of this site to the binding of 14-3-3, with concomitant loss of cell polarity and cell spreading in Madin-Darby canine kidney (MDCK) cells ${ }^{35}$. On the other hand, the kinases implicated in the phosphorylation of T340 and T360 have not been identified, although GSK3 $\beta$ has been suggested to indirectly influence the phosphorylation of these two sites, based on the observation that $\mathrm{LiCl}$, a potent and specific inhibitor of GSK3 $\beta$, significantly reduces binding of $14-3-3$ to IRSp53 $3^{36}$. Two other kinases, LRRK $2^{65}$ and $\mathrm{PKD}^{66}$, have been indirectly implicated in changes in T340 phosphorylation, although none has been shown to directly phosphorylate this site. Interestingly, like AMPK-related kinases, PKD works hand-in-hand with 14-3-3 in other 
cytoskeleton regulatory pathways. Thus, PKD-mediated phosphorylation of cortactin stimulates 14-3-3 binding, which interferes with cortactin's role as a stabilizer of Arp2/3 complexmediated actin branches ${ }^{67}$. PDK also phosphorylates the cofilin phosphatase slingshot-1L (SSH1L) to generate a 14-3-3-binding motif in SSH1L, which enhances the phosphorylation of cofilin and inhibits directed cell migration ${ }^{68}$. In the future it would be important to conclusively identify the kinases involved in IRSp53 regulation at 14-3-3-binding sites, and understand how they synergize with AMPK-related kinases to influence IRSp53's numerous activities and diverse interactions with downstream cytoskeletal effectors.

\section{Methods}

Protein cloning. For Co-IPs and protein purification, full-length IRSp53 (UniProt: Q9UQB8-4) was PCR-extended to include C-terminal FLAG- or STREP-affinity tags and cloned into vector pEGFP-Cl using the NheI and SalI restriction sites. IRSp53 mutations T340A, T360A, S366A, and S519C were introduced using the QuickChange II XL mutagenesis kit (Agilent Technologies, Santa Clara, CA). The ${ }^{452} \mathrm{SSST}^{455}$ to ${ }^{452} \mathrm{AAAA}^{455}$ mutation was generated by overlap PCR (primers listed in Supplementary Table 3). For the expression of wild-type/mutant IRSp53 heterodimers, the first and second genes were cloned respectively into the NheI/EcoRI and BamHI/NotI restriction sites of a modified vector pEGFP-C1 (containing a unique NotI restriction site in the multi-cloning site), carrying an internal ribosomal entry site (IRES) that was amplified from the plasmid pEF1a-IRES-Neo (Addgene, Cambridge, MA) and inserted into the EcoRI/BamHI restriction sites. This strategy allowed for the translation of two open reading frames from a single messenger RNA. For the expression of 2xFLAG-Eps8, full-length human Eps8 (UniProt: Q12929) was cloned into vector pEGFP-C1 and an oligonucleotide encoding for the 2xFLAG tag (MDYKDHDGDYKDDDDKG) was inserted between the NheI and EcoRI sites.

Mammalian cell protein expression and purification. For the expression of natively phosphorylated IRSp53-FLAG used in the FRET sensor assay, HEK293T (HEK293T/17) cells (ATCC; CRL-11268; Manassas, VA) were grown in DMEM GlutaMAX medium (Thermo Fisher Scientific, Waltham, MA) supplemented with 5\% FBS and antibiotic-antimycotic to $\sim 40 \%$ confluence in $15-\mathrm{cm}$ plates and transiently transfected with $10 \mu \mathrm{g}$ plasmid DNA in $30 \mu \mathrm{L}$ of a $1 \mathrm{mg} \mathrm{mL}^{-1}$ polyethylenimine (PEI) solution (Sigma-Aldrich Corporation, St. Louis, MO). After $48 \mathrm{~h}$ expression, IRSp53 phosphorylation was increased by serum-depriving cells for $2 \mathrm{~h}$. Cells were then washed and incubated in PBS with the addition of $0.1 \mu \mathrm{M}$ Calyculin A (Cell Signaling Technology, Danvers, MA) and 1x Halt Phosphatase Inhibitor Cocktail (Thermo Fisher Scientific) for $15 \mathrm{~min}$ at $37^{\circ} \mathrm{C}$. Cells were harvested, pelleted, and resuspended in lysis buffer comprised of PBS supplemented with $1 \mathrm{mM}$ EDTA, 5\% glycerol, $1 \%$ Triton X100, 1 mM PMSF, $0.1 \mu \mathrm{M}$ Calyculin A, $1 \mathrm{x}$ cOmplete protease inhibitor cocktail (Sigma-Aldrich Corporation) and 1x Halt Phosphatase Inhibitor Cocktail. Cells were incubated in lysis buffer on ice for $30 \mathrm{~min}$ and freeze-thawed three times. Insoluble cellular components and nuclei were removed by centrifugation at $12,000 \times g$ for $10 \mathrm{~min}$. Clarified lysates were incubated with $1 \mathrm{~mL}$ anti-FLAG antibody resin (Thermo Fisher Scientific) for $2 \mathrm{~h}$ at $4{ }^{\circ} \mathrm{C}$ and washed with 20 column volumes of lysis buffer (without Triton X100). Endogenous 14-3-3 was removed by washing the resin with five column volumes of wash buffer (PBS, $1 \mathrm{mM}$ EDTA, 5\% glycerol, 1x cOmplete protease inhibitor cocktail, and 1x Phosphatase Inhibitor Cocktail) supplemented with $200 \mathrm{mM}$ Sites 2,3 peptide. The excess peptide was removed with 10 column volumes of wash buffer, and IRSp53 was eluted with wash buffer supplemented with $300 \mathrm{mM} \mathrm{NaCl}$ and $0.5 \mathrm{mg} \mathrm{mL}^{-1} 3 \mathrm{xFLAG}$ peptide (Thermo Fisher Scientific). The fraction of phosphorylated IRSp53 able to bind 14-3-3 was then isolated by loading onto a 14-3-30-affinity column, consisting of MBP-tagged 14-3-30 (see expression below) bound to an amylose resin. The bound IRSp53 was eluted using $200 \mu \mathrm{M}$ Sites 2,3 peptide, and dialyzed into $25 \mathrm{mM}$ Tris- $\mathrm{HCl} \mathrm{pH} 8.0,150 \mathrm{mM} \mathrm{NaCl}, 5 \%$ glycerol and $1 \mathrm{mM}$ DTT. The protein was then incubate for $30 \mathrm{~min}$ at $4^{\circ} \mathrm{C}$ with $200 \mu \mathrm{L}$ of Macro-Prep High S cation exchange resin (Bio-Rad Laboratories, Hercules, CA). The resin was washed with 10 column volumes of dialysis buffer, followed by five column volumes of dialysis buffer supplemented with $200 \mathrm{mM} \mathrm{NaCl}$. IRSp53 was eluted with $500 \mu \mathrm{L}$ of $25 \mathrm{mM}$ HEPES pH 7.5, $500 \mathrm{mM} \mathrm{NaCl}, 1 \mathrm{mM} \mathrm{MgCl} 2,5 \%$ glycerol.

The construct 2xFLAG-Eps8 was transfected and expressed in HEK293T cells in a similar manner, except cells were not serum-starved or treated with phosphatase inhibitors. Cell lysis and FLAG-affinity purification were also performed as described above, except phosphatase inhibitors were not added to the buffer. 2xFLAG-Eps8 was eluted with wash buffer supplemented with $0.2 \mathrm{mg} \mathrm{mL}^{-1}$ 3xFLAG peptide and dialyzed against a buffer consisting of $25 \mathrm{mM}$ MES pH 6.7, $75 \mathrm{mM} \mathrm{NaCl}, 5 \%$ glycerol and $1 \mathrm{mM}$ DTT. The protein was concentrated and additionally purified using a Macro-Prep High S resin as above.
E. coli protein expression and purification. Human 14-3-30 (UniProt: P27348) was expressed in E. coli BL21(DE3) cells using the IMPACT expression vector pTYB11 (New England BioLabs, Ipswich, MA), carrying a chitin-binding domain (CBD) for affinity purification and an intein domain for self-cleavage of the affinity tag after purification. Cells were grown in TB medium, supplemented with $100 \mathrm{mg}$ $\mathrm{mL}^{-1}$ ampicillin at $37^{\circ} \mathrm{C}$. Protein expression was induced with $1 \mathrm{mM}$ IPTG at $18^{\circ} \mathrm{C}$ for $12 \mathrm{~h}$. Cells were homogenized in $25 \mathrm{mM}$ HEPES pH 7.5, $500 \mathrm{mM} \mathrm{NaCl}$, $1 \mathrm{mM}$ EDTA, $4 \mathrm{mM}$ benzamidine hydrochloride, and $1 \mathrm{mM}$ PMSF, and lysed using a microfluidizer (Microfluidics, Newton, MA). Lysates were clarified by centrifugation at 20,000 $\times g$ for $30 \mathrm{~min}$ and the supernatant was loaded onto the chitin affinity resin (New England BioLabs). The affinity tag was removed by self-cleavage of the intein domain induced by incubation with $50 \mathrm{mM}$ DTT overnight at room temperature. Proteins were eluted from the column in $25 \mathrm{mM}$ HEPES pH 7.5, 50 $\mathrm{mM} \mathrm{NaCl}$, and $1 \mathrm{mM}$ DTT. A final size exclusion chromatography purification step was performed on a Superdex 200 gel-filtration column (GE Healthcare, Little Chalfont, UK) in $25 \mathrm{mM}$ HEPES pH 7.5, $50 \mathrm{mM} \mathrm{NaCl}$ and $1 \mathrm{mM}$ DTT. The protein was concentrated to $\sim 60 \mathrm{mg} \mathrm{mL}^{-1}$ using an ultracentrifugation filter (EMD Millipore, Burlington, MA). 14-3-30 was also expressed as a maltose-binding protein (MBP) fusion in E. coli BL21(DE3). Cells were grown and lysed as described above, except cells were supplemented with $2 \mathrm{mg} \mathrm{mL}^{-1}$ glucose during growth. MBP-14-3-30 was loaded onto an amylose resin, and the resin was washed with 10 column volumes of a buffer consisting of $25 \mathrm{mM}$ HEPES pH 7.5, $300 \mathrm{mM}$ $\mathrm{NaCl}$ and $1 \mathrm{mM}$ DTT. MBP-14-3-30 was eluted in $25 \mathrm{mM}$ HEPES pH 7.5, $50 \mathrm{mM}$ $\mathrm{NaCl}, 1 \mathrm{mM}$ DTT, and $10 \mathrm{mM}$ maltose. The protein was additionally purified by size exclusion chromatography and concentrated as described above. Human $\mathrm{Cdc} 42$ (residues 1-178) constitutively active mutant G12V was also expressed in $E$. coli BL21(DE3) cells as above, using the IMPACT expression vector pTYB11 as we have described ${ }^{10}$.

Synthetic IRSp53 phospho-peptides. Site 2, Site 3, Site 4, Site 5, Sites 2,3, and Sites 2,4 IRSp53 phospho-peptides (Supplementary Table 1) were synthesized with $\mathrm{N}$-terminal acetylation and C-terminal amidation by Dr. Paul Leavis (Tufts University). The identity and purity of the peptides was verified by HPLC and mass spectrometry (Supplementary Figure 2). Site 1 and Sites 1,2 peptides were synthesized and purified by Neo Scientific (Cambridge, MA). Lyophilized peptides were dissolved at a concentration of $\sim 10 \mathrm{mM}$ and dialyzed extensively against $20 \mathrm{mM}$ HEPES pH 7.5, $50 \mathrm{mM} \mathrm{NaCl}, 1 \mathrm{mM} \mathrm{MgCl}$, and $0.1 \mathrm{mM}$ TCEP. The concentration of the peptides was determined using a fluorescence essay using fluorescamine-labeled peptide aliquots ${ }^{69}$.

Western blotting. Proteins were transferred from SDS-acrylamide gels onto immun-blot ${ }^{\circledast}$ PVDF membrane (Bio-Rad, Hercules, California) and blocked using Tris buffered saline (TBS) containing $0.1 \%$ Tween 20 (TBS-T) supplemented with $5 \%$ BSA prior to incubation with appropriate dilutions of primary antibodies (see below) for $4 \mathrm{~h}$ at $4{ }^{\circ} \mathrm{C}$. Excess primary antibody was removed by washing three times with TBS-T, and the blots were then incubated with appropriate dilutions of secondary antibodies (see below) for $30 \mathrm{~min}$ at room temperature. After removing excess secondary antibody by washing with TBS-T, the blots were developed by incubating with ECL2 reagent (Thermo Fisher Scientific) for 5 min and imaged using a G:Box fluorescent and chemiluminescent gel imager (Syngene, Frederick, MD). The relative abundance of proteins was determined using the program Fiji (https://imagej.net/Fiji) by densitometry analysis. All the blots presented in the figures were prepared from the unmodified blots shown in Supplementary Figure 9.

Antibodies. Mouse monoclonal anti-IRSp53 (sc-136470; 1:500 dilution), rabbit polyclonal anti-pan-14-3-3 (sc-629; 1:200 dilution), mouse monoclonal anti- $\beta$ actin (sc-47778; 1:1,000), mouse monoclonal GAPDH (sc-32233; 1:1,000 dilution), and rabbit polyclonal anti-FLAG-OctA (sc-807; 1:200 dilution) antibodies were purchased from Santa Cruz Biotechnology (Dallas, TX). The mouse monoclonal anti-E-cadherin antibody (610181; 1:1,000 dilution) was purchased from BD Biosciences (San Jose, CA). HRP-linked anti-mouse and anti-rabbit IgG (7076 S and $7074 \mathrm{~S} ; 1: 10,000$ dilution) were purchased from Cell Signaling Technologies. HRP-conjugated Streptavidin (N100; 1:5,000) was purchased from Thermo Fisher Scientific.

IRSp53 FRET. We have previously described the overall design and data collection strategy of the IRSp53 FRET sensor expressed in E. coli cells ${ }^{10}$. A critical modification here was the expression of the sensor protein ( $\left(\mathrm{PISp} 53_{\mathrm{FS}}\right.$ ) in mammalian HEK293T cells, which together with limited starvation ensures phosphorylation at 14-3-3-binding sites (see above). Briefly, IRSp53 contains four endogenous cysteine residues, but only one (C230 within the BAR domain) is accessible to labeling with fluorescence probes, as determined previously by mass spectrometry ${ }^{10}$. We introduced a second reactive cysteine near the C-terminus by site-directed mutagenesis (S519C mutant). To ensure that most of the donor probes are coupled to acceptor probes, we first under-labeled the protein with the donor fluorescein maleimide (Thermo Fisher Scientific), using a 0.9:1 molar ratio of probe to IRSp53 dimer for $30 \mathrm{~min}$ at $4{ }^{\circ} \mathrm{C}$. A two-fold molar excess of the acceptor rhodamine malemide (Thermo Fisher Scientific) was then added for $1 \mathrm{~h}$. The same batch of probes was used for all the labeling reactions of this study. Unreacted excess probes were 
removed by two successive passages through Zeba Spin Desalting Columns (Thermo Fisher Scientific), equilibrated in $25 \mathrm{mM}$ HEPES pH 7.5, $150 \mathrm{mM} \mathrm{NaCl}$, $1 \mathrm{mM} \mathrm{MgCl} 2,5 \%$ glycerol and $1 \mathrm{mM}$ DTT. The extent of labeling was determined by comparing the protein concentration to the concentration of the dyes, estimated from the peak absorbance of the fluorophores, using the extinction coefficients $\varepsilon(492 \mathrm{~nm})=83,000 \mathrm{~cm}^{-1} \mathrm{M}^{-1}$ for fluorescein-5-maleimide and $\varepsilon(542 \mathrm{~nm})=$ $91,000 \mathrm{~cm}^{-1} \mathrm{M}^{-1}$ for tetramethylrhodamine-5-maleimide. The IRSp53 dimer to total dye ratio was determined to be $\sim 1: 4$, indicating that the two reactive cysteine residues on each subunit of the IRSp53 dimer were labeled. Steady-state fluorescence emission spectra were recorded at $20^{\circ} \mathrm{C}$ upon titration of GNPPNP-Cdc42 (G12V mutant), Eps8 and/or 14-3-30 into a solution containing pIRSp53 $3_{\mathrm{FS}}$ (for each titration, the concentrations of the proteins are given in the legend to Fig. 5) using a Cary Eclipse Fluorescence Spectrophotometer (Agilent Technologies). The experiments were performed in $25 \mathrm{mM}$ Tris- $\mathrm{HCl} \mathrm{pH} 7.5,150 \mathrm{mM} \mathrm{NaCl}, 1 \mathrm{mM}$ $\mathrm{MgCl}_{2}, 5 \%$ glycerol, and $0.1 \mathrm{mM}$ TCEP. The excitation was set at $493 \mathrm{~nm}(10-\mathrm{nm}$ slit width) and the emission spectra were recorded at $520 \mathrm{~nm}$ (10-nm slit width). Titration curves were fit using the IGOR Pro 7 data analysis software (WaveMetrics, www.wavemetrics.com) to either a non-cooperative binding function $F$ ([ligand $])=$ $F_{\max } \times$ [ligand $] \times\left(K_{\mathrm{d}}+\right.$ [ligand $\left.]\right)^{-1}$ or a cooperative binding function with nonspecific binding $F([$ ligand $])=F_{\max } \times[\text { ligand }]^{n} \times\left(K_{\mathrm{d}}{ }^{n}+[\text { ligand }]^{n}\right)^{-1}+\mathrm{NS} \times$ [ligand] (as indicated), where $n$ is the Hill coefficient and NS is a constant nonspecific fit parameter. Best fits were selected on the basis of residual analysis.

Peptide FRET. The Site 2 and Sites 2,4 peptides were fluorescently labeled at the amidated C-terminus. Both peptides were labeled with the donor probe Alexa Fluor 488 NHS Ester, whereas the Site 2 peptide was additionally labeled with the acceptor probe Alexa Fluor 568 NHS Ester (Thermo Fisher Scientific). The labeling reaction was performed at $25^{\circ} \mathrm{C}$ for $1 \mathrm{~h}$ in $20 \mathrm{mM} \mathrm{MES} \mathrm{pH} 6.8$ and $50 \mathrm{mM} \mathrm{NaCl}$ and at a peptide concentration of $5 \mathrm{mg} \mathrm{mL}^{-1}$ with a 2 -fold molar excess of fluorescent dye. The labeling reaction was stopped with the addition of a 10-fold molar excess of hydroxylamine (Sigma-Aldrich Corporation). Labeled peptides were separated from any remaining unlabeled peptide using a Symmetry300 $\mathrm{C}_{18}$ reverse phase HPLC column (Waters Corporation, Milford, MA). The peptides were lyophilized, resuspended in $100 \mathrm{mM}$ HEPES $\mathrm{pH} 7.5$, and dialyzed against $25 \mathrm{mM}$ HEPES pH 7.5, $50 \mathrm{mM} \mathrm{NaCl}$ and $1 \mathrm{mM} \mathrm{MgCl}_{2}$. The donor-labeled peptides were pre-incubated with 14-3-30 at a 1:1 molar ratio (i.e., one peptide per 14-3-30 dimer). The acceptor-labeled Site 2 peptide (at $5 \mu \mathrm{M}$ concentration) was then titrated into the preformed complexes. The steady-state fluorescence emission spectra were recorded at $25^{\circ} \mathrm{C}$, with an excitation wavelength of $495 \mathrm{~nm}$ (5-nm slit width) and an emission wavelength of $520 \mathrm{~nm}$ (5-nm slit width).

Size exclusion chromatography-multi-angle light scattering. $14-3-3 \theta$ (100 $\mu \mathrm{l}$ at $4 \mathrm{mg} \mathrm{mL}^{-1}$ ) was pre-incubated for $2 \mathrm{~h}$ at $20^{\circ} \mathrm{C}$ with either the singly-phosphorylated Site 2 peptide at 1:2 molar ratio (one 14-3-30 dimer per two Site 2 peptides) or the doubly-phosphorylated Sites 2,4 peptide at 1:1 molar ratio (one 14-3-30 dimer per one Sites 2,4 peptide). The complexes were loaded onto a TSKgel SuperSW2000 column (Tosoh Corporation, Tokyo, Japan) connected in-line to a DAWN HELEOS MALS detector and an Optilab rEX (Wyatt Technology Corporation, Santa Barbara, CA) refractive index detector for mass analysis. Molecular masses were calculated with the program Astra (Wyatt Technology Corporation).

Phosphoproteomics. Coomassie-stained gels were cut into $1 \times 1 \mathrm{~mm}$ pieces, destained with $50 \%$ methanol and $1.25 \%$ acetic acid, reduced with $5 \mathrm{mM}$ DTT, and alkylated with $20 \mathrm{mM}$ iodoacetamide. The pieces were then washed with $20 \mathrm{mM}$ ammonium bicarbonate and dehydrated with acetonitrile. Proteins were proteolyzed overnight at $37^{\circ} \mathrm{C}$ with the addition of $5 \mathrm{ng} \mathrm{mL}^{-1}$ trypsin (Promega, Madison, WI) in $20 \mathrm{mM}$ ammonium bicarbonate. Peptides were extracted with $0.3 \%$ trifluoroacetic acid, followed by a $50 \%$ acetonitrile/water solution. Tryptic peptides were separated by reverse phase HPLC chromatography using a $75 \mu \mathrm{m}$ i. d. $\times 25 \mathrm{~cm}$ Acclaim PepMap nano LC column and eluted peptides were analyzed by LC-MS/MS on a Q Exactive HF mass spectrometer (Thermo Fisher Scientific). The mass spectrometer was set to repetitively scan the mass-to-charge ratio in the range 300-1400 (resolution of 240,000), followed by data-dependent MS/MS scans (resolution of 15,000 ) on the twenty most abundant ions, with a minimum automatic gain control (AGC) target of 1e4. Dynamic exclusion was enabled with a repeat count of 1 and a repeat duration of $30 \mathrm{~s}$. The Fourier Transform Mass Spectrometry (FTMS) full scan AGC target value was set to 3e6, while MSn AGC was 1e5. The MSn injection time was set to $160 \mathrm{~ms}$, and microscans were not averaged. FT preview mode charge state screening and monoisotopic precursor selection were all enabled, with rejection of unassigned and $1+$ charge states.

Database searching was performed using the Proteome Discoverer software v1.4 (Thermo Fisher Scientific), and searched against the UniProt human database appended with common contaminants using the SEQUEST algorithm. SEQUEST was searched with a fragment ion mass tolerance of $0.02 \mathrm{Da}$ and a parent ion tolerance of $10 \mathrm{ppm}$. Carbamidomethyl of cysteine was specified as a fixed modification. Methionine oxidation, N-terminal acetylation, and serine phosphorylation were specified as variable modifications. The program Scaffold v4.6.1 (Proteome Software Inc., Portland, OR) was used to validate MS/MS based peptide and protein identifications. Peptide identifications were accepted if they could be established at greater than $95.0 \%$ probability by the Scaffold Local FDR algorithm. Protein identifications were accepted if they could be established at greater than $95.0 \%$ probability and contained at least one identified peptide. Protein probabilities were assigned by the Protein Prophet algorithm ${ }^{70}$. Proteins that contained similar peptides and could not be differentiated based on MS/MS analysis alone were grouped to satisfy the principles of parsimony. Proteins sharing significant peptide evidence were grouped into clusters. The localization of the phosphorylation sites was validated using the Ascore algorithm ${ }^{71}$ in Scaffold PTM v3.2.0.

Isothermal titration calorimetry. ITC measurements were performed on a VPITC apparatus (MicroCal, Northampton, MA). Proteins and phospho-peptides were dialyzed side-by-side against $20 \mathrm{mM}$ HEPES, pH 7.5, $150 \mathrm{mM} \mathrm{NaCl}, 1 \mathrm{mM}$ $\mathrm{MgCl}_{2}$, and $0.1 \mathrm{mM}$ TCEP (ITC buffer). The buffer was replaced three times over a period of two days of dialysis. Singly-phosphorylated peptides (at $100 \mu \mathrm{M}$ ) and doubly-phosphorylated peptides (at $50 \mu \mathrm{M})$ in the syringe were titrated into 14-3$3 \theta($ at $10 \mu \mathrm{M})$ in the ITC cell of total volume $1.44 \mathrm{~mL}$. All the experiments were carried out at $20^{\circ} \mathrm{C}$. Titrations consisted of $10 \mu \mathrm{L}$ injections, lasting for $20 \mathrm{~s}$, with an interval of $5 \mathrm{~min}$ between injections. The heat of binding was corrected for the heat of injection, determined from either titrations of peptides into buffer (for the singly-phosphorylated peptides) or from the heat of injection at the end of the titration after reaching steady-state (for the less abundant doubly-phosphorylated peptides). Each experiment was repeated three times. The data were analyzed using the program Origin (OriginLab, Northampton, MA). Best fits were selected on the basis of residual analysis. The integrated heats from all the binding reactions fit well to a single-site binding model.

Crystallography. 14-3-30 was concentrated to $34 \mathrm{mg} \mathrm{mL}^{-1}$ in $20 \mathrm{mM}$ HEPES pH 7.5, $50 \mathrm{mM} \mathrm{NaCl}, 1 \mathrm{mM} \mathrm{MgCl}_{2}$, and $1 \mathrm{mM}$ DTT. Singly- and doublyphosphorylated IRSp53 peptides were pre-incubated with 14-3-30 at a molar ratio of $2.5: 1$ and $0.6: 1$, respectively, for $5 \mathrm{~min}$ at $25^{\circ} \mathrm{C}$. Crystals were obtained at $18^{\circ} \mathrm{C}$ using the hanging-drop vapor-diffusion method. A typical $2-\mu \mathrm{L}$ hanging drop consisted of a 1:1 (v/v) mixture of the well solution and the 14-3-30/peptide complexes. For the crystallization of the singly-phosphorylated peptide complexes (Site 2, Site 3, and Site 4), the well solution consisted of 13-18\% PEG 3350, $150 \mathrm{mM}$ magnesium formate and 4\% TFE. Crystals appeared after 1 day and were flashfrozen after a brief transfer into a cryo-solution containing 11\% PEG 400 and $11 \%$ PEG 1000 added to the crystallization buffer. For the crystallization of the doublyphosphorylated IRSp53 peptide complexes (Sites 2,3 and Sites 3,4), the well solution consisted of 13-15\% PEG 3350 and $100 \mathrm{mM} \mathrm{CaCl}_{2}$. Crystals grew over a period of three days and were slowly equilibrated into a cryo-solution containing $11 \%$ PEG 400 and 11\% PEG 1000 over the course of 1 day. All the X-ray diffraction datasets were collected at $100 \mathrm{~K}$ and on three different sources (as indicated in Supplementary Table 2): beamline 17-ID (APS, Argonne), beamline A1 (MacCHESS, Cornell University), and our home source (Bruker X8 Prospector, Billerica, MA). The datasets collected at synchrotron beamlines were indexed and scaled with the program HKL2000 (HKL Research) and the datasets collected at our home source were processed using the Bruker program SAINT (v8.34 A). The structures were determined by molecular replacement, using as a search model PDB entry 2BTP (excluding from this model the bound peptide). Model building and refinement were performed with the programs $\mathrm{Coot}^{72}$ and Phenix ${ }^{73}$, respectively. Figures of the structure were prepared with the program PyMOL (http://www. pymol.org/).

Membrane fractionation. HEK293T cells grown in $15-\mathrm{cm}$ plates were transfected with either IRSp53-FLAG or M234-FLAG. After $48 \mathrm{~h}$ expression, cells were incubated for $2 \mathrm{~h}$ in fresh growth media, which either contained or lacked $10 \%$ serum. Cells were then washed and incubated in PBS supplemented with $0.1 \mu \mathrm{M}$ Calyculin A and 1x Halt Phosphatase Inhibitor Cocktail for $15 \mathrm{~min}$ on ice. Each cell plate was harvested, pelleted, and resuspended in $250 \mu \mathrm{L}$ fractionation buffer consisting of 20 mM HEPES-KOH pH 7.4, $10 \mathrm{mM} \mathrm{KCl,} 2$ mM $\mathrm{MgCl}_{2}, 1 \mathrm{mM}$ EDTA, 1 mM EGTA, $1 \mathrm{mM}$ DTT, $1 \mathrm{mM}$ PMSF, $0.1 \mu \mathrm{M}$ Calyculin A, 1x cOmplete protease inhibitor cocktail and 1x Halt Phosphatase Inhibitor Cocktail, before passing through a 27 gauge needle 10 times. Ruptured cells were incubated on ice for $20 \mathrm{~min}$ and the nuclei were removed by centrifugation at $720 \times g$ for $5 \mathrm{~min}$. Mitochondria were removed from the supernatant by centrifugation at $10,000 \times g$ for $5 \mathrm{~min}$, and the resulting supernatant was separated into cytosolic and membrane fractions by ultracentrifugation at $100,000 \times g$ for $1 \mathrm{~h}$. The cytosolic fraction was transferred to a fresh tube, and the membrane fraction was resuspended in fractionation buffer by passing several times through a 25 -gauge needle. The membrane fraction was pelleted and resuspended two more times before resuspending the final membrane pellet in $250 \mu \mathrm{L}$ TBS supplemented with $0.1 \%$ SDS, $1 \mathrm{mM}$ PMSF, $0.1 \mu \mathrm{M}$ Calyculin A, 1x cOmplete protease inhibitor cocktail and 1x Halt Phosphatase Inhibitor Cocktail. The cytosolic and membrane fractions were verified by blotting using the cytosolic marker GAPDH and the plasma membrane marker E-cadherin. The relative abundance of IRSp53 was determined using the program Fiji by densitometry analysis of western blots that were transferred from SDS-acrylamide gels, loaded with equal volumes of cytosolic and membrane fractions for each condition. The abundance of IRSp53 in the cytosolic fraction was weighted according to the combined abundance of $\beta$-actin and GAPDH, whereas the membrane-bound 
fraction was weighted according to the abundance of E-cadherin. All the experiments were performed in triplicate, and from three independent transfections.

Statistical methods. The statistical significance of the measurements was determined using the program Prism v8.0.1 (GraphPad Software, Inc.), using the unpaired two-sided Student's $t$-test. A $p$-value $\leq 0.05$ was considered significant.

Reporting summary. Further information on experimental design is available in the Nature Research Reporting Summary linked to this article.

\section{Data availability}

The coordinates and structure factors of the five structures of complexes of 14-3-30 with singly- and doubly-phosphorylated IRSp53 peptides have been deposited in the Protein Data Bank (PDB) with accession codes 6BCR (Site 2), 6BCY (Site 3), 6BD1 (Site 4), 6BQT (Sites 2,3) and 6BD2 (Sites 2,4). A reporting summary for this Article is available as a Supplementary Information file. All other data supporting the findings of this study are available from the corresponding author upon request.

Received: 1 June 2018 Accepted: 17 December 2018

Published online: 29 January 2019

\section{References}

1. Kessels, M. M. \& Qualmann, B. Different functional modes of BAR domain proteins in formation and plasticity of mammalian postsynapses. J. Cell. Sci. 128, 3177-3185 (2015).

2. Nishimura, T., Morone, N. \& Suetsugu, S. Membrane re-modelling by BAR domain superfamily proteins via molecular and non-molecular factors. Biochem. Soc. Trans. 46, 379-389 (2018).

3. Miki, H., Yamaguchi, H., Suetsugu, S. \& Takenawa, T. IRSp53 is an essential intermediate between Rac and WAVE in the regulation of membrane ruffling. Nature 408, 732-735 (2000).

4. Krugmann, S. et al. Cdc42 induces filopodia by promoting the formation of an IRSp53:Mena complex. Curr Biol 11, 1645-1655 (2001).

5. Suetsugu, S. et al. Optimization of WAVE2 complex-induced actin polymerization by membrane-bound IRSp53, PIP(3), and Rac. J. Cell. Biol. 173, 571-585 (2006)

6. Disanza, A. et al. Regulation of cell shape by Cdc42 is mediated by the synergic actin-bundling activity of the Eps8-IRSp53 complex. Nat. Cell Biol. 8 1337-1347 (2006).

7. Scita, G., Confalonieri, S., Lappalainen, P. \& Suetsugu, S. IRSp53: crossing the road of membrane and actin dynamics in the formation of membrane protrusions. Trends Cell Biol 18, 52-60 (2008).

8. Goh, W. I. et al. mDial and WAVE2 proteins interact directly with IRSp53 in filopodia and are involved in filopodium formation. J. Biol. Chem. 287, 4702-4714 (2012).

9. Disanza, A. et al. CDC42 switches IRSp53 from inhibition of actin growth to elongation by clustering of VASP. EMBO J. 32, 2735-2750 (2013).

10. Kast, D. J. et al. Mechanism of IRSp53 inhibition and combinatorial activation by Cdc42 and downstream effectors. Nat. Struct. Mol. Biol. 21, 413-422 (2014).

11. Bockmann, J., Kreutz, M. R., Gundelfinger, E. D. \& Bockers, T. M. ProSAP/ Shank postsynaptic density proteins interact with insulin receptor tyrosine kinase substrate IRSp53. J. Neurochem. 83, 1013-1017 (2002).

12. Choi, J. et al. Regulation of dendritic spine morphogenesis by insulin receptor substrate 53, a downstream effector of Racl and Cdc42 small GTPases. J. Neurosci. 25, 869-879 (2005)

13. Sawallisch, C. et al. The insulin receptor substrate of $53 \mathrm{kDa}$ (IRSp53) limits hippocampal synaptic plasticity. J. Biol. Chem. 284, 9225-9236 (2009).

14. Chazeau, A. et al. Nanoscale segregation of actin nucleation and elongation factors determines dendritic spine protrusion. EMBO J. 33, 2745-2764 (2014).

15. Chung, W. et al. Social deficits in IRSp53 mutant mice improved by NMDAR and mGluR5 suppression. Nat Neurosci 18, 435-443 (2015).

16. Kang, J., Park, H. \& Kim, E. IRSp53/BAIAP2 in dendritic spine development, NMDA receptor regulation, and psychiatric disorders. Neuropharmacology 100, 27-39 (2016).

17. Soltau, M., Richter, D. \& Kreienkamp, H. J. The insulin receptor substrate IRSp53 links postsynaptic shank1 to the small G-protein cdc42. Mol. Cell. Neurosci. 21, 575-583 (2002).

18. Lim, K. B. et al. The Cdc42 effector IRSp53 generates filopodia by coupling membrane protrusion with actin dynamics. J. Biol. Chem. 283, 20454-20472 (2008).
19. Funato, Y. et al. IRSp53/Eps8 complex is important for positive regulation of Rac and cancer cell motility/invasiveness. Cancer Res. 64, 5237-5244 (2004).

20. Oikawa, T. et al. IRSp53 mediates podosome formation via VASP in NIH-Src cells. PLoS. One. 8, e60528 (2013).

21. Nakagawa, H. et al. IRSp53 is colocalised with WAVE2 at the tips of protruding lamellipodia and filopodia independently of Mena. J. Cell. Sci. 116, 2577-2583 (2003)

22. Abou-Kheir, W., Isaac, B., Yamaguchi, H. \& Cox, D. Membrane targeting of WAVE2 is not sufficient for WAVE2-dependent actin polymerization: a role for IRSp53 in mediating the interaction between Rac and WAVE2. J. Cell. Sci. 121, 379-390 (2008).

23. Fujiwara, T., Mammoto, A., Kim, Y. \& Takai, Y. Rho small G-proteindependent binding of mDia to an Src homology 3 domain-containing IRSp53/ BAIAP2. Biochem. Biophys. Res. Commun. 271, 626-629 (2000).

24. Soltau, M. et al. Insulin receptor substrate of $53 \mathrm{kDa}$ links postsynaptic shank to PSD-95. J. Neurochem. 90, 659-665 (2004).

25. Barilari, M. \& Dente, L. The neuronal proteins CIPP, Cypin and IRSp53 form a tripartite complex mediated by PDZ and SH3 domains. Biol. Chem. 391, 1169-1174 (2010)

26. Segal, D., Dhanyasi, N., Schejter, E. D. \& Shilo, B. Z. Adhesion and fusion of muscle cells are promoted by filopodia. Dev. Cell. 38, 291-304 (2016).

27. Chauhan, B. K. et al. Cdc42- and IRSp53-dependent contractile filopodia tether presumptive lens and retina to coordinate epithelial invagination. Development 136, 3657-3667 (2009).

28. Liu, P. S., Jong, T. H., Maa, M. C. \& Leu, T. H. The interplay between Eps8 and IRSp53 contributes to Src-mediated transformation. Oncogene 29, 3977-3989 (2010)

29. Toma, C. et al. Association study of six candidate genes asymmetrically expressed in the two cerebral hemispheres suggests the involvement of BAIAP2 in autism. J. Psychiatr. Res. 45, 280-282 (2011).

30. Fromer, M. et al. De novo mutations in schizophrenia implicate synaptic networks. Nature 506, 179-184 (2014).

31. Ribases, M. et al. Case-control study of six genes asymmetrically expressed in the two cerebral hemispheres: association of BAIAP2 with attention-deficit/ hyperactivity disorder. Biol. Psychiatry 66, 926-934 (2009).

32. Prevost, C. et al. IRSp53 senses negative membrane curvature and phase separates along membrane tubules. Nat. Commun. 6, 8529 (2015).

33. Saarikangas, J. et al. Molecular mechanisms of membrane deformation by IBAR domain proteins. Curr. Biol. 19, 95-107 (2009).

34. Mattila, P. K. et al. Missing-in-metastasis and IRSp53 deform PI(4,5)P2-rich membranes by an inverse BAR domain-like mechanism. J. Cell. Biol. 176, 953-964 (2007).

35. Cohen, D., Fernandez, D., Lazaro-Dieguez, F. \& Musch, A. The serine/ threonine kinase Parlb regulates epithelial lumen polarity via IRSp53mediated cell-ECM signaling. J. Cell. Biol. 192, 525-540 (2011).

36. Robens, J. M., Yeow-Fong, L., Ng, E., Hall, C. \& Manser, E. Regulation of IRSp53-dependent filopodial dynamics by antagonism between 14-3-3 binding and SH3-mediated localization. Mol. Cell. Biol. 30, 829-844 (2010).

37. Cornell, B. \& Toyo-Oka, K. 14-3-3 proteins in brain development: neurogenesis, neuronal migration and neuromorphogenesis. Front. Mol. Neurosci. 10, 318 (2017).

38. Mackie, S. \& Aitken, A. Novel brain 14-3-3 interacting proteins involved in neurodegenerative disease. Febs. J. 272, 4202-4210 (2005).

39. Jin, J. et al. Proteomic, functional, and domain-based analysis of in vivo 14-3-3 binding proteins involved in cytoskeletal regulation and cellular organization. Curr. Biol. 14, 1436-1450 (2004).

40. Bright, N. J., Thornton, C. \& Carling, D. The regulation and function of mammalian AMPK-related kinases. Acta Physiol. (Oxf.). 196, 15-26 (2009).

41. Banko, M. R. et al. Chemical genetic screen for AMPKalpha2 substrates uncovers a network of proteins involved in mitosis. Mol. Cell 44, 878-892 (2011).

42. Schaffer, B. E. et al. Identification of AMPK phosphorylation sites reveals a network of proteins involved in cell invasion and facilitates large-scale substrate prediction. Cell. Metab. 22, 907-921 (2015).

43. Kahn, B. B., Alquier, T., Carling, D. \& Hardie, D. G. AMP-activated protein kinase: ancient energy gauge provides clues to modern understanding of metabolism. Cell. Metab. 1, 15-25 (2005).

44. Hornbeck, P. V. et al. PhosphoSitePlus, 2014: mutations, PTMs and recalibrations. Nucleic Acids Res. 43, D512-D520 (2015).

45. Johnson, C. et al. Bioinformatic and experimental survey of 14-3-3-binding sites. Biochem. J. 427, 69-78 (2010).

46. Yaffe, M. B. et al. The structural basis for 14-3-3:phosphopeptide binding specificity. Cell 91, 961-971 (1997).

47. Obsil, T., Ghirlando, R., Anderson, D. E., Hickman, A. B. \& Dyda, F. Two 14 3-3 binding motifs are required for stable association of Forkhead transcription factor FOXO4 with 14-3-3 proteins and inhibition of DNA binding. Biochemistry 42, 15264-15272 (2003). 
48. Kostelecky, B., Saurin, A. T., Purkiss, A., Parker, P. J. \& McDonald, N. Q. Recognition of an intra-chain tandem 14-3-3 binding site within PKCepsilon. EMBO Rep. 10, 983-989 (2009).

49. Molzan, M., Weyand, M., Rose, R. \& Ottmann, C. Structural insights of the MLF1/14-3-3 interaction. Febs. J. 279, 563-571 (2012).

50. Stevers, L. M. et al. Characterization and small-molecule stabilization of the multisite tandem binding between 14-3-3 and the R domain of CFTR. Proc. Natl Acad. Sci. USA 113, E1152-E1161 (2016).

51. Stevers, L. M. et al. Structural interface between LRRK2 and 14-3-3 protein. Biochem. J. 474, 1273-1287 (2017)

52. Sluchanko, N. N. et al. Structural basis for the interaction of a human small heat shock protein with the 14-3-3 universal signaling regulator. Structure 25, 305-316 (2017).

53. Petrvalska, O. et al. Structural Insight into the 14-3-3 protein-dependent Inhibition of Protein Kinase ASK1 (apoptosis signal-regulating kinase 1). J. Biol. Chem. 291, 20753-20765 (2016).

54. Gardino, A. K. \& Yaffe, M. B. 14-3-3 proteins as signaling integration points for cell cycle control and apoptosis. Semin. Cell. Dev. Biol. 22, 688-695 (2011).

55. Kleppe, R., Martinez, A., Doskeland, S. O. \& Haavik, J. The 14-3-3 proteins in regulation of cellular metabolism. Semin. Cell. Dev. Biol. 22, 713-719 (2011).

56. Gohla, A. \& Bokoch, G. M. 14-3-3 regulates actin dynamics by stabilizing phosphorylated cofilin. Curr. Biol. 12, 1704-1710 (2002).

57. Freeman, A. K. \& Morrison, D. K. 14-3-3 Proteins: diverse functions in cell proliferation and cancer progression. Semin. Cell. Dev. Biol. 22, 681-687 (2011).

58. Boudreau, A. et al. 14-3-3sigma stabilizes a complex of soluble actin and intermediate filament to enable breast tumor invasion. Proc. Natl Acad. Sci. USA 110, E3937-E3944 (2013).

59. Rosenberg, M. M. et al. Adenomatous polyposis coli plays a key role, in vivo, in coordinating assembly of the neuronal nicotinic postsynaptic complex. Mol. Cell. Neurosci. 38, 138-152 (2008).

60. Yaffe, M. B. How do 14-3-3 proteins work?-Gatekeeper phosphorylation and the molecular anvil hypothesis. FEBS Lett. 513, 53-57 (2002).

61. Reinhardt, H. C. \& Yaffe, M. B. Phospho-Ser/Thr-binding domains: navigating the cell cycle and DNA damage response. Nat. Rev. Mol. Cell Biol. 14, 563-580 (2013)

62. Stevers, L. M. et al. Modulators of 14-3-3 Protein-Protein Interactions. J. Med. Chem. 61, 3755-3778 (2018)

63. Oda, K. et al. Identification of BAIAP2 (BAI-associated protein 2), a novel human homologue of hamster IRSp53, whose SH3 domain interacts with the cytoplasmic domain of BAI1. Cytogenet. Cell Genet. 84, 75-82 (1999).

64. Madeira, F. et al. 14-3-3-Pred: improved methods to predict 14-3-3-binding phosphopeptides. Bioinformatics 31, 2276-2283 (2015).

65. Luerman, G. C. et al. Phosphoproteomic evaluation of pharmacological inhibition of leucine-rich repeat kinase 2 reveals significant off-target effects of LRRK-2-IN-1. J. Neurochem. 128, 561-576 (2014).

66. Franz-Wachtel, M. et al. Global detection of protein kinase D-dependent phosphorylation events in nocodazole-treated human cells. Mol. Cell. Proteom. 11, 160-170 (2012)

67. Eiseler, T., Hausser, A., De Kimpe, L., Van Lint, J. \& Pfizenmaier, K. Protein kinase D controls actin polymerization and cell motility through phosphorylation of cortactin. J. Biol. Chem. 285, 18672-18683 (2010).

68. Eiseler, T. et al. Protein kinase D1 regulates cofilin-mediated F-actin reorganization and cell motility through slingshot. Nat. Cell Biol. 11, 545-556 (2009).

69. Udenfriend, S. et al. Fluorescamine: a reagent for assay of amino acids, peptides, proteins, and primary amines in the picomole range. Science 178, 871-872 (1972).
70. Nesvizhskii, A. I., Keller, A., Kolker, E. \& Aebersold, R. A statistical model for identifying proteins by tandem mass spectrometry. Anal. Chem. $\mathbf{7 5}$, 4646-4658 (2003)

71. Beausoleil, S. A., Villen, J., Gerber, S. A., Rush, J. \& Gygi, S. P. A probabilitybased approach for high-throughput protein phosphorylation analysis and site localization. Nat. Biotechnol. 24, 1285-1292 (2006).

72. Emsley, P., Lohkamp, B., Scott, W. G. \& Cowtan, K. Features and development of Coot. Acta Crystallogr D. 66, 486-501 (2010).

73. Adams, P. D. et al. The Phenix software for automated determination of macromolecular structures. Methods 55, 94-106 (2011).

\section{Acknowledgements}

This research was supported by National Institutes of Health (NIH) grant R01 MH087950 to R.D. D.J.K. was in part supported by American Cancer Society grant PF13-033-01-DMC. We thank Paul Leavis (Tufts University) for the synthesis of some of the peptides used in this study. X-ray data collection at beamline X6A of the National Synchrotron Light Source was supported by NIH grant GM-0080 and DOE contract DEAC02-98CH10886. X-ray data collection at MacCHESS beamline A1 was supported by NSF grant DMR-1332208 and NIH grant GM-103485.

\section{Author contributions}

D.R.K. and R.D. participated equally in the experimental design and the preparation of the manuscript and figures. D.K. conducted most of the experiments, and R.D. participated in the determination of the crystal structures.

\section{Additional information}

Supplementary Information accompanies this paper at https://doi.org/10.1038/s41467 019-08317-8.

Competing interests: The authors declare no competing interests.

Reprints and permission information is available online at http://npg.nature.com/ reprintsandpermissions/

Journal peer review information: Nature Communications thanks the anonymous reviewers for their contribution to the peer review of this work. Peer reviewer reports are available.

Publisher's note: Springer Nature remains neutral with regard to jurisdictional claims in published maps and institutional affiliations.

Open Access This article is licensed under a Creative Commons Attribution 4.0 International License, which permits use, sharing, adaptation, distribution and reproduction in any medium or format, as long as you give appropriate credit to the original author(s) and the source, provide a link to the Creative Commons license, and indicate if changes were made. The images or other third party material in this article are included in the article's Creative Commons license, unless indicated otherwise in a credit line to the material. If material is not included in the article's Creative Commons license and your intended use is not permitted by statutory regulation or exceeds the permitted use, you will need to obtain permission directly from the copyright holder. To view a copy of this license, visit http://creativecommons.org/ licenses/by/4.0/.

(c) The Author(s) 2019 